\title{
Neutrino Oscillations in the Early Universe: A Real-Time Formulation
}

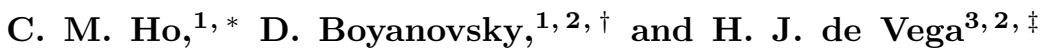 \\ ${ }^{1}$ Department of Physics and Astronomy, University of Pittsburgh, Pittsburgh, Pennsylvania 15260, USA \\ ${ }^{2}$ Observatoire de Paris, LERMA, Laboratoire Associé au CNRS UMR 8112, \\ 61, Avenue de l'Observatoire, 75014 Paris, France. \\ ${ }^{3}$ LPTHE, Université Pierre et Marie Curie (Paris VI) et Denis Diderot (Paris VII), \\ Laboratoire Associé au CNRS UMR 7589, Tour 24, \\ 5ème. étage, 4, Place Jussieu, 75252 Paris, Cedex 05, France
}

(Dated: November 21, 2018)

\begin{abstract}
Neutrino oscillations in the early Universe prior to the epoch of primordial nucleosynthesis is studied by implementing real time non-equilibrium field theory methods. We focus on two flavors of Dirac neutrinos, however, the formulation is general. We obtain the equations of motion for neutrino wavepackets of either chirality and helicity in the plasma allowing for $\mathrm{CP}$ asymmetry. Contributions non-local in space-time to the self-energy dominate over the asymmetry for $T \gtrsim 3-5 \mathrm{MeV}$ if the lepton and neutrino asymmetries are of the same order as the baryon asymmetry. We find a new contribution which cannot be interpreted as the usual effective potential. The mixing angles and dispersion relations in the medium depend on helicity. We find that resonant transitions are possible in the temperature range $10 \lesssim T \ll 100 \mathrm{MeV}$. Near a resonance in the mixing angle, the oscillation time scale in the medium as compared to the vacuum is slowed-down substantially for small vacuum mixing angle. The time scale of oscillations speeds-up for off resonance high energy neutrinos for which the mixing angle becomes vanishingly small. The equations of motion reduce to the familiar oscillation formulae for negative helicity ultrarelativistic neutrinos, but include consistently both the mixing angle and the oscillation frequencies in the medium. These equations of motion also allow to study the dynamics of right handed as well as positive helicity neutrinos.
\end{abstract}

PACS numbers: $13.15 .+\mathrm{g}, 12.15 .-\mathrm{y}, 11.10 . \mathrm{Wx}$

\section{INTRODUCTION}

Neutrinos have taken center stage in particle physics and have become a bridge between astrophysics, cosmology, particle physics and nuclear physics $1,2,3,4,45]$.

A wealth of experimental data have confirmed that neutrinos are massive and that different flavors of neutrinos can mix and oscillate 6, 7, 8, 9, 10, 11, 12] thus providing indisputable evidence for new physics beyond the Standard Model.

Neutrinos play a fundamental role in cosmology and astrophysics, and it is now widely accepted that resonant flavor oscillations due to the MSW effect in the sun provide a concrete explanation to the solar neutrino problem [5, 13, 14, 15].

Neutrino oscillations in extreme conditions of temperature and density are an important aspect of Big Bang Nucleosynthesis (BBN) and in the generation of the lepton asymmetry in the early Universe 4, 10, 16, 17, 18], as well as in the physics of core collapse supernovae 6 , 15, 20], and the formation, evolution and cooling of neutron stars [21, 22, 23].

While accelerator and reactor experiments measure the difference of the neutrino masses, high precision cosmological observations of the cosmic microwave background by WMAP combined with large scale structure suggest that the sum of the masses of all neutrino species is bound to be smaller than $1 \mathrm{eV}$ [24].

An important aspect of neutrino oscillations is lepton number violation, leading to the suggestion that the baryon asymmetry may actually originate in the lepton sector and the proposal that leptogenesis can be the main mechanism that explains the cosmological baryon asymmetry [25, 26, 27].

Neutrino propagation in a cold medium has been first studied in ref. [13] wherein the refractive index of electron neutrinos was computed. The early studies of neutrino propagation focused on the neutrino dispersion relations and damping rates in the temperature regime relevant for stellar evolution or big bang nucleosynthesis [17, 28]. Since

\footnotetext{
*Electronic address: cmho@phyast.pitt.edu

${ }^{\dagger}$ Electronic address: boyan@pitt.edu

${ }^{\ddagger}$ Electronic address: devega@lpthe.jussieu.fr
} 
then, the work has been extended to include leptons, neutrinos and nucleons in the medium [29]. The matter effects of neutrino oscillations in the early universe has been investigated in 30,31 .

Since the original study of neutrino oscillations in the sun [13, 14], neutrino oscillations are typically studied within the single particle quantum mechanical formulation. For two flavors the evolution Hamiltonian is simply that for a two-state system, where the off-diagonal terms lead to the mixing and oscillation phenomena. The medium properties are input in this formulation after computing the contributions from charged and neutral currents in a medium with leptons, neutrinos and hadrons or quarks. A conceptually similar approach underlies the kinetic treatment of oscillations in the early Universe wherein the dynamics is studied from the time evolution of a density matrix that generalizes the single particle description but that does not generally account for the subtle aspects of flavor Fock states addressed in ref [37, which introduce a hierarchy of time scales. Recently [33] a set of generalized semiclassical Boltzmann equations for the single particle distribution functions, supposedly applicable to neutrino transport in corecollapse supernovae have been proposed, but where the mixing term must be obtained separately from the underlying field theory. For a thorough discussion of the kinetic approach to mixing and relaxation and the approximations involved, the reader is referred to $17,32,33]$.

The dynamics of neutrino mixing in the presence of a background of neutrinos requires in general a full nonlinear treatment, which has so far been studied within a self-consistent single particle framework 34] in the form of approximate kinetic equations for the reduced density matrix 17, 32]. Such study revealed a wealth of novel nonlinear phenomena such as self-synchronization [34]. An approximate treatment of background neutrinos within the framework of equilibrium finite temperature field theory has also been proposed in ref. [35]. However, a consistent treatment must necessarily rely on a non-linear kinetic description, which has not yet been developed in the full field theory framework.

A calculation of the neutrino dispersion relations in a hot and dense medium implementing the techniques of quantum field theory at finite temperature was provided in ref. [28]. This treatment was extended in ref. [35] to study the propagation of mixed neutrinos of negative helicity in a neutrino background, up to lowest order in $g^{2} / M_{W}^{2}$. In ref. [36] the quantum fields for Dirac neutrinos propagating in a cold but dense medium and the dispersion relation for both chirality components were obtained, again considering the medium corrections to the dispersion relations up to order $g^{2} / M_{W}^{2}$.

The study of dynamics of neutrino mixing in the literature is mostly carried out within the framework of a single particle description, wherein the dynamical evolution is described in terms of an effective Hamiltonian for either a two or three level system (depending the number of flavors). However, a single particle formulation is inadequate in a hot and or dense medium where collective many body effects may be predominant. The main point in the above discussion is to highlight that there is a leap in the current approach to study neutrino oscillations in a medium: the result of a quantum field theory calculation of the index of refraction or effective potentials in the medium is input into a single particle quantum mechanical description of oscillations and mixing based on Bloch-type equations.

We have previously reported on a study of oscillations in a hot and/or dense neutrino gas directly from the underlying quantum field theory [37] in free field theory. Such study, even at the free field level revealed quantum interference phenomena and subtle many body aspects responsible for a hierarchy of time scales that cannot be captured within the single particle description. In particular, the subtle aspects of the Fock representation of the distribution function as well as interference and coherence phenomena, leading to widely different time scales has not been fully included in the treatments in ref. $17,32,33$.

More recently, novel collective neutrino excitations in the standard model (namely without masses and mixing) near the critical temperature were studied [38]. Collective phenomena requires a systematic and consistent treatment implementing the methods of quantum field theory at finite temperature and density. Furthermore, the real time dynamics of mixing and oscillations requires a non-equilibrium formulation of quantum field theory specially suited to study the real time evolution as an initial value problem [39]- 43 .

There are at least four fundamental reasons to study neutrinos in the early Universe at a deeper level: i) neutrino mixing may be the mechanism by which baryogenesis is a result of leptogenesis 25, 26, 27], ii) big bang nucleosynthesis is particularly sensitive to the spectrum and oscillations of neutrinos [18, 19, 30], iii) just like the cosmic microwave background, there is a cosmic neutrino background left over from the big bang, and iv) neutrinos masses and mixing are the most clear experimental confirmation of physics beyond the standard model. All of these reasons warrant a complete quantum field theory study of neutrinos in the early Universe.

\section{The goals of this article:}

The full quantum field theory treatment of neutrino mixing in hot and or dense media has not yet received the same level of attention as the more familiar single particle treatment, which however, is not suited when collective many body phenomena become relevant as is typically the case in extreme environments.

Previous quantum field theory studies [17, 28, 35, 36] address either the dispersion relations or mixing phenomena under restrictive approximations to lowest order in $g^{2} / M_{W}^{2}$. 
In this article we provide a systematic quantum field theory study of neutrino propagation and oscillations in the early Universe directly in real time. Because in the early Universe the lepton asymmetries are expected to be typically of the same order of the baryon asymmetry $\eta_{B} / \eta_{\gamma} \sim 10^{-9}$ a consistent description of neutrino propagation and oscillations requires to include corrections non-local in space-time of order $g^{2} / M_{W}^{4}$ in the dispersion relations 28] and mixing angles. We focus our study on the case of two flavors of Dirac neutrinos, taken to be the electron and muon neutrinos, this study can be generalized to more flavors or to Majorana-Dirac mass matrices without any conceptual difficulty. Our main goals are: i ) To provide a systematic and consistent study of the real time dynamics of neutrino oscillation and mixing directly in quantum field theory in conditions of temperature and lepton/neutrino asymmetries applicable to the early Universe prior to the nucleosynthesis era. This is achieved by formulating an initial value problem via linear response and implementing real time field theory methods at finite temperature and density. ii) To obtain the dispersion relations and in-medium mixing angles including the non local contributions from the neutrino self-energies up to order $g^{2} / M_{W}^{4}$. The one-loop self-energy in expanded to lowest order in $(\omega, k) / M_{W}$. We find a new contribution which cannot be interpreted as the usual effective potential. These contributions are necessary since the typical asymmetries in the early Universe are very small and these non-local (in space-time) contributions can be of the same order or larger than the local contributions.iii) To obtain the in-medium Dirac spinors for both helicities and study the evolution of oscillations and mixing for both helicity components directly in real time. iv) Two different temperature regimes are studied in detail: i) $m_{e} \ll T \ll m_{\mu}$, ii) $m_{e}, m_{\mu} \ll T \ll M_{W}$. The first regime is just prior to big bang nucleosynthesis. Lepton and hadron (proton and neutrons in nuclear statistical equilibrium) or quark asymmetries are included in the one-loop self-energy. We assess in detail the temperature and energy regime for which a resonance in the mixing angle is available in the medium. The second temperature regime is above the QCD phase transition and we include two flavor of (light quarks) with their respective asymmetries. In this regime the mixing angle becomes small. In both cases we also study the mixing and oscillations of positive helicity as well as right handed neutrinos, which are typically neglected in the literature. We also obtain the loop corrections to the oscillation frequencies thereby providing a complete description of oscillation and mixing that includes corrections to both the mixing angle and the oscillation frequencies. v) We obtain general oscillation formulae derived directly from the real time evolution in quantum field theory. These formulae reveal the limit in which the usual quantum mechanical single particle description is reliable as well as the corrections to them.

Main approximations: Since our study relies on a one-loop self-energy computation including leptons and neutrinos, the inclusion of a neutrino background must necessarily imply some approximations for consistency.

We do not yet consider absorptive contributions, which in the temperature regime studied here are of two-loop order, postponing the study of the interplay between oscillations and relaxation to a forthcoming article.

Since we obtain the non-local (in space-time) contributions from the one-loop self energy we must address the issue of the neutrino propagators in the neutral current contributions. Because of mixing, the neutrino propagator in the flavor basis, in which the weak interactions are diagonal, does not correspond to the propagation of mass eigenstates and in principle the non-equilibrium propagators obtained in ref. [37] must be used. The question of equilibration of a neutrino gas with mixing is one of time scales: the weak interactions are diagonal in the flavor basis, therefore weak processes tend to equilibrate flavor neutrinos with a typical weak interaction relaxation rate at high temperature 28] $\Gamma \sim G_{F}^{2} T^{5}$. Oscillations, on the other hand mix flavors and tend to redistribute flavor neutrinos into mass eigenstates of energy $E$ on a time scale $\tau_{\text {osc }} \sim E / \delta M^{2}$. Combined fitting of the solar and KamLAND data yield [45] $\left|\delta M^{2}\right| \approx 7.9 \times 10^{-5}(\mathrm{eV})^{2}$, therefore considering $E \sim T$, we find $\Gamma \tau_{\text {osc }} \sim 10(0.1 \mathrm{~T} / \mathrm{MeV})^{6}$. This comparison of time scales suggests that for $T \gtrsim 10 \mathrm{MeV}$ neutrinos are equilibrated as flavor eigenstates. Flavor eigenstates created at local weak interaction vertices will reach thermal equilibrium on time scales far shorter than those required for oscillations into mass eigenstates for temperatures larger than $\sim 10 \mathrm{MeV}$. Since in a loop integral the typical momenta are of order $T$, and assuming the validity of this estimate, we consider the neutrino propagators in the neutral current self-energy loop to be diagonal in the flavor basis, massless and in thermal equilibrium.

For temperatures $T \lesssim 10 \mathrm{MeV}$ and certainly below freeze out $T<1 \mathrm{MeV}$ a full kinetic description that includes oscillations and expansion [17, 30] is required. The study of the kinetic equations will be the subject of forthcoming work. In this article we restrict our study to the temperature regime $T \gtrsim 10 \mathrm{MeV}$.

We also assume that the lepton and neutrino asymmetries are of the same order as the baryon asymmetry, namely $L_{i}=\left(n_{i}-\bar{n}_{i}\right) / n_{\gamma} \sim 10^{-9}$. For a relativistic species the asymmetry is proportional to $\xi_{i}\left(1+\xi_{i}^{2} / \pi^{2}\right)$ with $\xi_{i} \equiv \mu_{i} / T$, therefore under this assumption $\xi_{i} \sim 10^{-9}$ and we can safely neglect the contribution to the chemical potential in the non-local (in space-time) terms of order $g^{2} / M_{W}^{4}$.

Brief summary of main results: The main results obtained in this article are: i) We obtain the equation of motion in real time for initially prepared wavepackets of neutrinos. Both chiralities and helicities are treated on equal footing. The self-energy is expanded up to lowest order in the frequency and momentum yielding nonlocal contributions to the equations of motion. These happen to be larger than those from the lepton and neutrino asymmetries for $T \gtrsim 5-10 \mathrm{MeV}$. ii) We studied two different temperature regimes: $m_{e} \ll T \ll m_{\mu}$ within which we show that there is the possibility of resonant oscillations of test neutrinos, and $m_{e}, m_{\mu} \ll T \ll M_{W}$ within which 
the mixing angle for active neutrinos effectively vanishes. For $T \sim 10 \mathrm{MeV}$ resonant flavor oscillations occur for neutrino energies in the few MeV range. iii) Mixing angles in the medium not only depend on energy but also on helicity. The dispersion relations of propagating neutrinos in the medium also depend on helicity. Assuming that the lepton and quark asymmetries are of the same order as the baryon asymmetry in the early Universe, the non-local (in space-time) terms in the self-energies dominate over the asymmetry for typical energies of neutrinos in the plasma for $T \gtrsim 3-5 \mathrm{MeV}$. iv)The oscillation time scale in the medium is slowed-down near the resonance, becoming substantially longer than in the vacuum for small vacuum mixing angle. For high energy neutrinos off-resonance the mixing angle becomes vanishingly small and the oscillation time scale speeds-up as compared to the vacuum. $\mathbf{v}$ ) The equations of motion reduce to the familiar oscillation formulae for ultrarelativistic negative helicity neutrinos, but they consistently include the mixing angles and the oscillation frequencies in the medium. We obtain general oscillation formula for either chirality and helicity. These equations also describe the oscillation dynamics of right handed neutrinos, which, while suppressed as consequence of the small masses, are not sterile.

The article is organized as follows: in section II we obtain the equations of motion for initially prepared neutrino wavepackets by implementing the methods of non-equilibrium field theory and linear response. In section III we obtain the one-loop self-energy contributions from charged and neutral currents. Section IV] is devoted to obtaining the dispersion relations, mixing angles and oscillation time scales in the medium and a study of the possibility of resonances. In section $\nabla$ we study the real time evolution of neutrino wavepackets as an initial value problem. Section VI presents our conclusions, summarizes our results and presents some conjectures and further questions. The detailed calculation of the self-energy is presented in an appendix.

\section{EFFECTIVE DIRAC EQUATION FOR NEUTRINO PROPAGATION IN A MEDIUM}

The propagation of a neutrino in a medium is determined by the effective Dirac equation which includes the selfenergy corrections. Its solution yields the real time evolution as an initial value problem. The correct framework to study the dynamics is the real time formulation of field theory in terms of the closed-time-path integral 39]-[43]. In this section we implement this method combined with linear response to obtain the effective equation of motion for an expectation value of the neutrino field. The main concept in this approach is the following, consider coupling an external c-number Grassman source to the neutrino field and switching this source adiabatically up to time $t=0$. This source induces an expectation value of the neutrino field, after switching-off the external source at $t=0$, the expectation value evolves in time as a solution of the effective Dirac equation in the medium with the initial condition determined by the source term.

The main ingredient in this program is the retarded self-energy which enters in the effective Dirac equation. The real-time formulation of field theory directly leads to causal and retarded equations of motion. It is important to highlight the difference with the S-matrix approach which describes transition amplitudes from in to out states, the real time formulation yields the equations of motion for an expectation value and these are fully causal 39 ]- 43 ].

The self-energy is obtained in the unitary gauge in which only the correct physical degrees of freedom contribute and is manifestly unitary [4]. Previous calculations of the neutrino self-energy in covariant gauges (one of which is the unitary gauge) have proven that although the self-energy does depend on the gauge parameter, the dispersion relations are gauge-invariant 29$]$.

As mentioned above we restrict our discussion to the case of two flavors of Dirac neutrinos, namely the electron and muon neutrinos. The subtle $C P$ violating phases associated with the case of three active neutrinos will not be considered here. However, the method can be generalized to three active neutrinos, sterile neutrinos or even Majorana neutrinos without any conceptual difficulty and will be postponed for further discussion elsewhere.

For Dirac neutrinos, mixing and oscillations can be implemented by a minimal extension of the standard model adding a Dirac mass matrix to the standard model Lagrangian which is off-diagonal in the flavor basis. The relevant part of the Lagrangian density is given by

$$
\mathcal{L}=\mathcal{L}_{\nu}^{0}+\mathcal{L}_{W}^{0}+\mathcal{L}_{Z}^{0}+\mathcal{L}_{C C}+\mathcal{L}_{N C}
$$

where $\mathcal{L}_{\nu}^{0}$ is the free field neutrino Lagrangian minimally modified to include a Dirac mass matrix

$$
\mathcal{L}_{\nu}^{0}=\bar{\nu}_{a}\left(i \not \partial \delta_{a b}-M_{a b}\right) \nu_{b}
$$

with $a, b$ being the flavor indexes. For two flavors of Dirac neutrinos the mass matrix $M_{a b}$ is given by

$$
\mathbf{M}=\left(\begin{array}{ll}
m_{e e} & m_{e \mu} \\
m_{e \mu} & m_{\mu \mu}
\end{array}\right),
$$


$\mathcal{L}_{W, Z}^{0}$ are the free field lagrangian densities for the vector bosons in the unitary gauge, namely

$$
\begin{aligned}
\mathcal{L}_{W}^{0} & =-\frac{1}{2}\left(\partial_{\mu} W_{\nu}^{+}-\partial_{\nu} W_{\mu}^{+}\right)\left(\partial^{\mu} W^{-\nu}-\partial^{\nu} W^{-\mu}\right)+M_{W}^{2} W_{\mu}^{+} W^{-\mu} \\
\mathcal{L}_{Z}^{0} & =-\frac{1}{4}\left(\partial_{\mu} Z_{\nu}-\partial_{\nu} Z_{\mu}\right)\left(\partial^{\mu} Z^{\nu}-\partial^{\nu} Z^{\mu}\right)+\frac{1}{2} M_{Z}^{2} Z_{\mu} Z^{\mu}
\end{aligned}
$$

and the charged and neutral current interaction lagrangian densities are given by

$$
\begin{gathered}
\mathcal{L}_{C C}=\frac{g}{\sqrt{2}}\left[\bar{\nu}_{a} \gamma^{\mu} L l_{a} W_{\mu}^{+}+\bar{l}_{a} \gamma^{\mu} L \nu_{a} W_{\mu}^{-}\right] \\
\mathcal{L}_{N C}=\frac{g}{2 \cos \theta_{w}}\left[\bar{\nu}_{a} \gamma^{\mu} L \nu_{a} Z_{\mu}+\bar{f}_{a} \gamma^{\mu}\left(g_{a}^{V}-g_{a}^{A} \gamma^{5}\right) f_{a} Z_{\mu}\right] .
\end{gathered}
$$

where $L=\left(1-\gamma^{5}\right) / 2$ is the left-handed chiral projection operator, $g^{V, A}$ are the vector and axial vector couplings for quarks and leptons, $l$ stands for leptons and $f$ generically for the fermion species with neutral current interactions.

For two flavors, the diagonalization of the free field Dirac Lagrangian for neutrinos, (II.2) is achieved by a unitary transformation to mass eigenstates. Considering, flavor and mass doublets respectively

$$
\left(\begin{array}{l}
\nu_{e} \\
\nu_{\mu}
\end{array}\right) \quad, \quad\left(\begin{array}{l}
\nu_{1} \\
\nu_{2}
\end{array}\right)
$$

related by unitary transformation

$$
\left(\begin{array}{c}
\nu_{e} \\
\nu_{\mu}
\end{array}\right)=U\left(\begin{array}{c}
\nu_{1} \\
\nu_{2}
\end{array}\right)
$$

with the unitary transformation given by the $2 \times 2$ matrix

$$
U=\left(\begin{array}{cc}
\cos \theta & \sin \theta \\
-\sin \theta & \cos \theta
\end{array}\right)
$$

where $\theta$ is the vacuum mixing angle.

In the basis of mass eigenstates $\left(\nu_{1}, \nu_{2}\right)$ the mass matrix $M_{a b}$ becomes diagonal

$$
\left(\begin{array}{cc}
M_{1} & 0 \\
0 & M_{2}
\end{array}\right)
$$

The elements $m_{e e}, m_{\mu \mu}$ and $m_{e \mu}$ in the mass matrix (11.3) are related to the vacuum mixing angle $\theta$ and masses of the propagating mass eigenstates $M_{1}$ and $M_{2}$ as follows

$$
m_{e e}=C^{2} M_{1}+S^{2} M_{2} ; m_{\mu \mu}=S^{2} M_{1}+C^{2} M_{2} ; m_{e \mu}=-\left(M_{1}-M_{2}\right) C S,
$$

where $C=\cos \theta$ and $S=\sin \theta$.

For later convenience, we introduce

$$
\bar{M}=\frac{M_{1}+M_{2}}{2} ; \delta M^{2}=M_{1}^{2}-M_{2}^{2}
$$

The current value for the average of the vacuum masses obtained by WMAP 24] and oscillation parameters from the combined fitting of the solar and KamLAND data are [45]:

$$
\bar{M} \approx 0.25(\mathrm{eV}) ;\left|\delta M^{2}\right| \approx 7.9 \times 10^{-5}(\mathrm{eV})^{2} ; \tan ^{2} \theta \approx 0.40 .
$$

For these values of the masses and more generally if there is an almost degeneracy in the hierarchy of neutrino masses the ratio

$$
\frac{\left|\delta M^{2}\right|}{\bar{M}^{2}} \ll 1
$$

The smallness of this ratio in the nearly degenerate case will lead to important simplifications. 

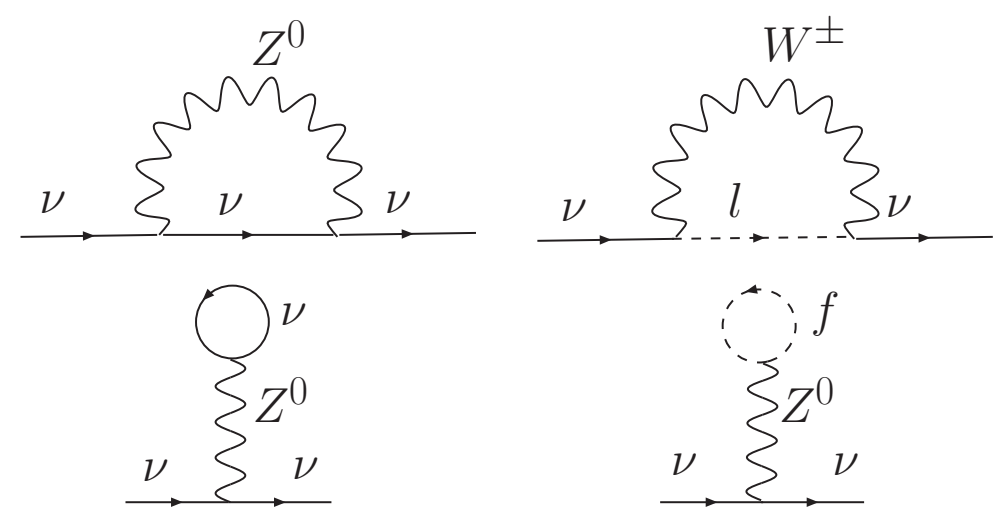

FIG. 1: One-loop diagrams contributing to the neutrino self-energy.

Our goal is to obtain the effective Dirac equation for neutrinos propagating in the medium and extract the inmedium mixing angles, propagation frequencies and the wave functions of the propagating modes in the medium. The real-time effective Dirac equation in the medium is derived from linear response by implementing the methods of non-equilibrium quantum field theory described in [43].

Following this approach, we introduce an external Grassmann-valued source that couples linearly to the neutrino field via the lagrangian density

$$
\mathcal{L}_{S}=\bar{\nu}_{a} \eta_{a}+\bar{\eta}_{a} \nu_{a}
$$

whence the total lagrangian density is given by $\mathcal{L}+\mathcal{L}_{S}$. The external source induces an expectation value for the neutrino field which, in turn, obeys the effective equation of motion with self-energy modifications from the thermal medium [43].

To study the dynamics of the system, it is the expectation values rather than the in-out S-matrix elements that are necessary [40]. This requires a generating function for the real-time correlation functions. Denoting generically by $\Phi$ the fields (fermions, or gauge bosons), a path integral representation of this generating functional is given by

$$
\mathcal{Z}\left[j^{+}, j^{-}\right]=\int D \Phi^{+} D \Phi^{-} e^{i \int\left(\mathcal{L}\left[\Phi^{+}, j^{+}\right]-\mathcal{L}\left[\Phi^{-}, j^{-}\right]\right)},
$$

and the path integrations over the fields $\Phi^{ \pm}$will be taken along the forward $(+)$and backward $(-)$time branches, in the presence of the sources $j^{ \pm}[39]$ - [33]. Here, the sources $j^{ \pm}$are coupled linearly to the fields $\Phi^{ \pm}$and thus the real-time correlation functions can be obtained from the functional derivatives of this generating functional with respect to these sources. Functional derivatives with respect to $j^{+}$and $j^{-}$give the time-ordered and anti-time-ordered correlation functions respectively.

The sources $j^{ \pm}$are introduced to compute the real-time correlation functions and will be set to zero after the calculations. However, the external Grassman source $\eta$ and hence the expectation value induced to the neutrino field will remain the same along both time branches $( \pm)$. For further discussions on the general method, see references 39, 41, 42, 43].

The equation of motion for the expectation value of the neutrino field induced by the external Grassman source is derived by shifting the field

$$
\nu_{a}^{ \pm}=\psi_{a}+\Psi_{a}^{ \pm} \quad, \quad \psi_{a}=\left\langle\nu_{a}^{ \pm}\right\rangle \quad, \quad\left\langle\Psi_{a}^{ \pm}\right\rangle=0,
$$

and imposing $\left\langle\Psi_{a}^{ \pm}\right\rangle=0$ order by order in the perturbation theory [41, 42, 43]. Carrying out this implementation up to one-loop order, we find the following equation of motion

$$
\left(i \not \partial \delta_{a b}-M_{a b}+\Sigma_{a b}^{t a d} L\right) \psi_{b}(\vec{x}, t)+\int d^{3} x^{\prime} \int d t^{\prime} \Sigma_{a b}^{r e t}\left(\vec{x}-\vec{x}^{\prime}, t-t^{\prime}\right) \psi_{b}\left(\vec{x}^{\prime}, t^{\prime}\right)=-\eta_{a}(\vec{x}, t),
$$

where $\Sigma_{a b}^{r e t}\left(\vec{x}-\vec{x}^{\prime}, t-t^{\prime}\right)$ is the real-time retarded self-energy given by the exchange one-loop contributions, the first two diagrams displayed in Fig. 1 and $\Sigma_{a b}^{t a d} L$ is the tadpole contribution from the last two diagrams in Fig. 1 The expectation value of the neutrino field in the medium describes a beam or wave-packet of test neutrinos, namely these are neutrinos that are injected in the medium, for example by the decay of a neutron or any other heavy particle, but 
have not (yet) thermalized with the plasma. This is precisely the manner in which linear response leads to a study of real-time phenomena.

Due to the translational invariance of the medium in thermal equilibrium, the retarded self-energy is simply a function of $\vec{x}-\vec{x}^{\prime}$ and $t-t^{\prime}$. Hence, the equation can be written in frequency and momentum space by introducing the space-time Fourier transform of the expectation value and the source

$$
\psi_{a}(\vec{x}, t)=\frac{1}{\sqrt{V}} \sum_{\vec{k}} \int d \omega \psi_{a}(\vec{k}, \omega) e^{i \vec{k} \cdot \vec{x}} e^{-i \omega t} \quad, \quad \eta_{a}(\vec{x}, t)=\frac{1}{\sqrt{V}} \sum_{\vec{k}} \int d \omega \eta_{a}(\vec{k}, \omega) e^{i \vec{k} \cdot \vec{x}} e^{-i \omega t} .
$$

Furthermore, due to the rotational invariance of the thermal medium, implies that all physical quantities depend on $|\vec{k}| \equiv k$.

We have argued in the introduction that for temperatures $T \gtrsim 10 \mathrm{MeV}$ the relaxation via the weak interaction is faster than the time scales of (vacuum) oscillations. The validation of this assumption requires a deeper study of the interplay between neutrino oscillations and damping processes in the medium including the two-loop self-energy, which will be the subject of a forthcoming article. Assuming the validity of this estimate, the neutrinos in the loop are in thermal equilibrium as flavor eigenstates and at the temperatures of interest we consider them massless. Under this assumption, the self-energy is diagonal in the flavor basis

As a result, the effective Dirac equation for neutrino oscillations in the medium is obtained as

$$
\left[\left(\gamma^{0} \omega-\vec{\gamma} \cdot \vec{k}\right) \delta_{a b}-M_{a b}+\Sigma_{a b}^{t a d} L+\Sigma_{a b}(\omega, k) L\right] \psi_{b}(\omega, k)=-\eta_{a}(\omega, k),
$$

with

$$
\Sigma(\omega, k)=\Sigma_{W}(\omega, k)+\Sigma_{Z}(\omega, k) ; \Sigma^{t a d}=\Sigma^{\nu_{e} t a d}+\Sigma^{\nu_{\mu} t a d}+\Sigma^{f t a d},
$$

where $\Sigma^{f t a d}$ is the total tadpole contribution from all fermions other than neutrinos in the loop.

The details of the calculation of the self-energy are presented in the Appendix.

\section{ONE-LOOP SELF-ENERGIES}

\section{A. Charged and Neutral Currents}

From Eqs. A.28)-A.32) in the Appendix, we found that the self-energies $\Sigma_{W, Z}(\omega, k)$ can be written in the dispersive form

$$
\Sigma_{W, Z}(\omega, k)=\int \frac{d k_{0}}{\pi} \frac{\operatorname{Im} \Sigma_{W}\left(k_{0}, k\right)}{k_{0}-\omega-i \epsilon} .
$$

with

$$
\begin{aligned}
& \operatorname{Im} \Sigma_{W}\left(k_{0}, k\right)=\frac{g^{2} \pi}{2} \int \frac{d^{3} p}{(2 \pi)^{3}} \frac{1}{4 W_{q} \omega_{p}}\left\{\left[1-N_{f}\left(\omega_{p}\right)+N_{b}\left(W_{q}\right)\right] \varnothing(\vec{p}, \vec{q}) \delta\left(k_{0}-\omega_{p}-W_{q}\right)+\right. \\
& +\left[1-\bar{N}_{f}\left(\omega_{p}\right)+N_{b}\left(W_{q}\right)\right] \varnothing(-\vec{p},-\vec{q}) \delta\left(k_{0}+\omega_{p}+W_{q}\right)+\left[N_{f}\left(\omega_{p}\right)+N_{b}\left(W_{q}\right)\right] \varnothing(\vec{p},-\vec{q}) \delta\left(k_{0}-\omega_{p}+W_{q}\right)+ \\
& \left.+\left[\bar{N}_{f}\left(\omega_{p}\right)+N_{b}\left(W_{q}\right)\right] \varnothing(-\vec{p}, \vec{q}) \delta\left(k_{0}+\omega_{p}-W_{q}\right)\right\}
\end{aligned}
$$

where we have defined

$$
\begin{aligned}
& Q^{\mu}(\vec{p}, \vec{q})=p^{\mu}+2 q^{\mu} \frac{W_{q} \omega_{p}-\vec{q} \cdot \vec{p}}{M_{W}^{2}} \\
& q^{\mu}=\left(W_{q}, \vec{k}-\vec{p}\right) \quad, \quad \omega_{p}=\sqrt{|\vec{p}|^{2}+m_{f}^{2}} \quad, \quad W_{q}=\sqrt{|\vec{k}-\vec{p}|^{2}+M_{W}^{2}}
\end{aligned}
$$

The corresponding contribution from neutral currents can be obtained from the above expression by setting

$$
\frac{g}{\sqrt{2}} \rightarrow \frac{g}{2 \cos \theta_{w}} \quad, \quad M_{W} \rightarrow M_{Z}=\frac{M_{W}}{\cos \theta_{w}}
$$


with $\theta_{w}$ being the Weinberg angle.

In the limit $T \ll M_{W, Z}$, the abundance of vector bosons is exponentially suppressed, hence we neglect the terms that feature $N_{b}\left(W_{q}\right)$. The imaginary part of the one-loop self-energy vanishes on the neutrino mass shell at one-loop level. A non-vanishing damping rate (non-vanishing imaginary part of the self-energy at the neutrino mass shell) at temperatures $T \ll M_{W}$ arises at two-loop level. Thus we focus solely on $\operatorname{Re} \Sigma_{W}\left(k_{0}, k\right)$ when studying the dispersion relation and propagation of neutrinos in the medium.

The form of $\operatorname{Im} \Sigma_{W}\left(k_{0}, k\right)$ suggests that $\operatorname{Re} \Sigma_{W}(\omega, k)$ can be written as

$$
\operatorname{Re} \Sigma_{W}(\omega, k)=\gamma^{0} \sigma_{W}^{0}(\omega, k)-\vec{\gamma} \cdot \widehat{\mathbf{k}} \sigma_{W}^{1}(\omega, k),
$$

where $\sigma_{W}^{0}(\omega, k)$ and $\sigma_{W}^{1}(\omega, k)$ can be obtained by taking traces on both sides.

Dropping the $T=0$ part and using the dispersive representation (III.1), we find that for any fermion $f$ in the loop,

$$
\begin{aligned}
& \sigma_{W}^{0}(\omega, k)=-\frac{g^{2}}{2} \int \frac{d^{3} p}{(2 \pi)^{3}} \frac{1}{4 W_{q} \omega_{p}}\{ N_{f}\left(\omega_{p}\right)\left[\frac{Q_{0}(\vec{p}, \vec{q})}{W_{q}+\omega_{p}-\omega}+\frac{Q_{0}(\vec{p},-\vec{q})}{\left.W_{q}-\omega_{p}+\omega\right)}\right] \\
&\left.-\bar{N}_{f}\left(\omega_{p}\right)\left[\frac{Q_{0}(\vec{p}, \vec{q})}{W_{q}+\omega_{p}+\omega}+\frac{Q_{0}(\vec{p},-\vec{q})}{W_{q}-\omega_{p}-\omega}\right]\right\}, \\
& \sigma_{W}^{1}(\omega, k)=-\frac{g^{2}}{2} \int \frac{d^{3} p}{(2 \pi)^{3}} \frac{1}{4 W_{q} \omega_{p}}\left\{\begin{array}{l}
N_{f}\left(\omega_{p}\right)\left[\frac{\widehat{\mathbf{k}} \cdot \vec{Q}(\vec{p}, \vec{q})}{W_{q}+\omega_{p}-\omega}+\frac{\widehat{\mathbf{k}} \cdot \vec{Q}(\vec{p},-\vec{q})}{W_{q}-\omega_{p}+\omega}\right] \\
\left.+\bar{N}_{f}\left(\omega_{p}\right)\left[\frac{\widehat{\mathbf{k}} \cdot \vec{Q}(\vec{p}, \vec{q})}{W_{q}+\omega_{p}+\omega}+\frac{\widehat{\mathbf{k}} \cdot \vec{Q}(\vec{p},-\vec{q})}{W_{q}-\omega_{p}-\omega}\right]\right\} .
\end{array}\right.
\end{aligned}
$$

In the thermalized medium with temperature $T$, the dominant loop momenta is of order $p \sim T$, therefore we neglect the neutrino masses since $T \gtrsim 3-5 \mathrm{MeV}$. The self-energy is expanded in a power series in the external frequency and momentum $\omega, k$, we refer to terms of the form $\omega / M_{W} ; k / M_{W}$ as non-local terms (in space-time) since they represent gradient expansions in configuration space. Furthermore, we neglect the contribution from leptons with masses $m_{f} \gg$ $T$ since these will be exponentially suppressed, but we calculate the self-energies up the order $\left(g^{2} / M_{W, Z}^{4}\right)\left(m_{f} / T\right)^{2}$ for leptons with masses $m_{f} \ll T$ and all higher order terms will be dropped. A straightforward but lengthy calculation gives

$$
\begin{aligned}
\sigma_{W}^{0}(\omega, k) & =-\frac{3 G_{F}}{\sqrt{2}}\left(n_{f}-n_{\bar{f}}\right)+\frac{7 \pi^{2}}{15 \sqrt{2}} \frac{G_{F} \omega T^{4}}{M_{W}^{2}}, \\
\sigma_{W}^{1}(\omega, k) & =-\frac{7 \pi^{2}}{45 \sqrt{2}} \frac{G_{F} k T^{4}}{M_{W}^{2}}\left[1-\frac{30}{7 \pi^{2}}\left(\frac{m_{f}}{T}\right)^{2}\right],
\end{aligned}
$$

where $G_{F}=\sqrt{2} \frac{g^{2}}{8 M_{W}^{2}}$ is the Fermi constant, and $n_{f}-n_{\bar{f}}$ is the particle-antiparticle number density difference for any fermion $f$ defined as

$$
n_{f}-n_{\bar{f}}=2 \int \frac{d^{3} p}{(2 \pi)^{3}}\left[N_{f}\left(\omega_{p}\right)-\bar{N}_{f}\left(\omega_{p}\right)\right] .
$$

The contribution to $\sigma_{W}^{1}(\omega, k)$ of order $g^{2} / M_{W}^{2}$ vanishes as a consequence of the isotropy of the equilibrium distribution functions. In calculating the non-local (in space-time) terms proportional to $\omega / M_{W} ; k / M_{W}$ we have neglected the chemical potentials under the assumption that all asymmetries (leptons and neutrinos) are of the same order as the baryon asymmetry, in which case $\mu / T \sim 10^{-9}$ where $\mu$ is the chemical potential for the corresponding species.

In ref. 28] the equivalent to $\sigma^{0}$ is quoted as the coefficient $b_{L}$, but no equivalent to $\sigma^{1}$ was provided there, this is a difference between our results and those of ref. [28]. The contribution $\sigma^{1}(\omega, k)$ is new and it cannot be identified with an "effective potential" (which is proportional to $\gamma^{0}$ ) and it will be a source of helicity dependence on frequencies and mixing angles which has not been appreciated in the literature.

To obtain the corresponding expressions for the neutral current interactions, we can simply apply the replacement eq. (III.4). The tadpole contributions have been previously obtained in ref. [28] where we refer the reader for more details. The result of the tadpole diagrams is summarized by the expression

$$
\Sigma^{f t a d}=\frac{2 G_{F} g^{V}}{\sqrt{2}}\left(n_{f}-n_{\bar{f}}\right) \gamma^{0},
$$

where the coefficient $g^{V}$ for the different species of leptons, hadrons and quarks are given in the table below. 


\begin{tabular}{|c|c|}
\hline Particles & $g^{V}$ \\
\hline$\nu_{e}, \nu_{\mu}, \nu_{\tau}$ & $\frac{1}{2}$ \\
\hline$e, \mu, \tau$ & $-\frac{1}{2}+2 \sin ^{2} \theta_{w}$ \\
\hline$p$ & $\frac{1}{2}-2 \sin ^{2} \theta_{w}$ \\
\hline$n$ & $-\frac{1}{2}$ \\
\hline$u, c, t$ & $\frac{1}{2}-\frac{4}{3} \sin ^{2} \theta_{w}$ \\
\hline$d, s, b$ & $-\frac{1}{2}+\frac{2}{3} \sin ^{2} \theta_{w}$ \\
\hline
\end{tabular}

Writing $\Sigma^{f t a d}(\omega, k)$ in the same form as (III.5), we obtain

$$
\sigma_{t a d}^{0}=\frac{2 G_{F} g^{V}}{\sqrt{2}}\left(n_{f}-n_{\bar{f}}\right) \quad, \quad \sigma_{t a d}^{1}=0 .
$$

and tadpoles with quark loops acquire an extra factor 3 from color. The tadpole contribution is proportional to $n_{f}-n_{\bar{f}}$, which is the signature of a $C P$ asymmetric medium. It is customary and convenient to express $n_{f}-n_{\bar{f}}$ in terms of its relative abundance to the photons in the universe. At any temperature $T$, the photon number density in the universe is given by

$$
n_{\gamma}=\frac{2}{\pi^{2}} \zeta(3) T^{3} .
$$

and the particle-antiparticle asymmetry for any fermion species $f$ is defined as

$$
L_{f}=\frac{n_{f}-n_{\bar{f}}}{n_{\gamma}} .
$$

The magnitude of the observed baryon asymmetry is $L_{B} \simeq 10^{-9}$. Since $B-L$ is conserved in the standard model, it is natural to expect that the lepton asymmetries should be of the same order as $L_{B}$. Although there is no a priori reason to expect the neutrino asymmetries to be of the same order, we will henceforth assume that all the lepton and neutrino asymmetries $L_{e}, L_{\mu}, L_{\nu_{e}}$ and $L_{\nu_{\mu}}$ are of the order of the baryon asymmetry $\sim 10^{-9}$.

The next step is to compute the contributions from the one-loop exchange diagrams. We focus on two different temperature regimes, $m_{e} \ll T \ll m_{\mu}$ in which only the electron is ultrarelativistic and $m_{e}, m_{\mu} \ll T \ll M_{W}$ in which both leptons are ultrarelativistic.

$$
\text { 1. } \mathbf{m}_{\mathbf{e}} \ll \mathbf{T} \ll \mathbf{m}_{\mu}
$$

This temperature limit is interesting because it is the energy scale right above BBN. The efficiency of BBN is sensitive to the amount of electron neutrinos which, in turn, depends on the detailed dynamics of neutrino oscillations. As it will be discussed below in detail, in the temperature regime $T \gtrsim 5 \mathrm{MeV}$ the non-local (in space-time) terms proportional to $\omega, k$ from the exchange diagrams (both charged and neutral currents) dominate the contributions of the lepton and neutrino asymmetries assuming all of them to be of order $10^{-9}$.

In the temperature limit with $m_{e} \ll T \ll m_{\mu}$, the contribution from $\mu$ leptons to the exchange one loop diagram is exponentially suppressed and we neglect it. Apart from electrons and neutrinos, the thermal background does contain protons and neutrons in nuclear statistical equilibrium since for $T \gtrsim 1 \mathrm{MeV}$ the weak interactions lead to equilibration on time scales shorter than the Hubble time scale via the reactions

$$
n \leftrightarrow p+e^{-}+\bar{\nu}_{e} \quad, \quad p+\bar{\nu}_{e} \leftrightarrow n+e^{+} \quad, \quad p+e^{-} \leftrightarrow n+\nu_{e} .
$$

In the basis of flavor eigenstates, the total one-loop self-energy contribution is of the form

$$
\operatorname{Re} \Sigma(\omega, k)=\left[\gamma^{0} \mathbb{A}(\omega)-\vec{\gamma} \cdot \widehat{\mathbf{k}} \mathbb{B}(k)\right] L
$$

where $\mathbb{A}(\omega, k)$ and $\mathbb{B}(\omega, k)$ are $2 \times 2$ diagonal matrices in the neutrino flavor basis given by

$$
\mathbb{A}(\omega)=\left(\begin{array}{cc}
A_{e}(\omega) & 0 \\
0 & A_{\mu}(\omega)
\end{array}\right) ; \quad \mathbb{B}(k)=\left(\begin{array}{cc}
B_{e}(k) & 0 \\
0 & B_{\mu}(k)
\end{array}\right),
$$


Extracting non-local terms (in space-time) up to $\mathcal{O}(\omega / T), \mathcal{O}(k / T)$ we find the following matrix elements,

$$
\begin{aligned}
A_{e}(\omega)= & -\frac{3 G_{F}}{\sqrt{2}} L_{e} n_{\gamma}+\frac{7 \pi^{2}}{15 \sqrt{2}} \frac{G_{F} \omega T^{4}}{M_{W}^{2}}-\frac{3 G_{F}}{2 \sqrt{2}} L_{\nu_{e}} n_{\gamma}+\frac{7 \pi^{2}}{30 \sqrt{2}} \frac{G_{F} \omega T^{4}}{M_{Z}^{2}} \\
& +\frac{G_{F}}{\sqrt{2}} L_{\nu_{e}} n_{\gamma}+\frac{G_{F}}{\sqrt{2}} L_{\nu_{\mu}} n_{\gamma}+\frac{2 G_{F}}{\sqrt{2}}\left(-\frac{1}{2}+2 \sin ^{2} \theta_{w}\right) L_{e} n_{\gamma} \\
& +\frac{2 G_{F}}{\sqrt{2}}\left(\frac{1}{2}-2 \sin ^{2} \theta_{w}\right) L_{p} n_{\gamma}-\frac{G_{F}}{\sqrt{2}} L_{n} n_{\gamma}, \\
A_{\mu}(\omega)= & -\frac{3 G_{F}}{2 \sqrt{2}} L_{\nu_{\mu}} n_{\gamma}+\frac{7 \pi^{2}}{30 \sqrt{2}} \frac{G_{F} \omega T^{4}}{M_{Z}^{2}} \\
& +\frac{G_{F}}{\sqrt{2}} L_{\nu_{e}} n_{\gamma}+\frac{G_{F}}{\sqrt{2}} L_{\nu_{\mu}} n_{\gamma}+\frac{2 G_{F}}{\sqrt{2}}\left(-\frac{1}{2}+2 \sin ^{2} \theta_{w}\right) L_{e} n_{\gamma} \\
& +\frac{2 G_{F}}{\sqrt{2}}\left(\frac{1}{2}-2 \sin ^{2} \theta_{w}\right) L_{p} n_{\gamma}-\frac{G_{F}}{\sqrt{2}} L_{n} n_{\gamma}, \\
B_{e}(k)= & -\frac{7 \pi^{2}}{45 \sqrt{2}} \frac{G_{F} k T^{4}}{M_{W}^{2}}\left[1-\frac{30}{7 \pi^{2}}\left(\frac{m_{e}}{T}\right)^{2}\right]-\frac{7 \pi^{2}}{90 \sqrt{2}} \frac{G_{F} k T^{4}}{M_{Z}^{2}}, \\
B_{\mu}(k)= & -\frac{7 \pi^{2}}{90 \sqrt{2}} \frac{G_{F} k T^{4}}{M_{Z}^{2}} .
\end{aligned}
$$

We purposely displayed the individual terms in the above expressions to highlight that the first line in $A_{e, \mu}(\omega)$ as well as the expressions for $B_{e, \mu}(k)$ arise from the exchange diagrams (the two top diagrams in fig 1 ), while the second and third lines in $A_{e, \mu}(\omega)$ arise from the tadpole diagrams (bottom two diagrams in fig 1 ). We have assumed that the flavor neutrinos are in thermal equilibrium and have consistently neglected neutrino masses. The correction term $\left(m_{e} / T\right)^{2}$ is displayed so that one can estimate the error incurred when this term is dropped for $T>>m_{e}$, the error is less than $1 \%$ for $T \gtrsim 5 \mathrm{MeV}$. In what follows we will neglect this contribution in the temperature range of interest for this section.

Charge neutrality requires that $L_{e}=L_{p}$, hence

$$
\Sigma^{e t a d}+\Sigma^{p t a d}=0 .
$$

Therefore, the expressions for $A_{e}$ and $A_{\mu}$ simplify to the following:

$$
\begin{aligned}
& A_{e}(\omega)=\frac{G_{F} n_{\gamma}}{\sqrt{2}}\left[-\mathcal{L}_{e}+\frac{7 \pi^{4}}{60 \zeta(3)} \frac{\omega T}{M_{W}^{2}}\left(2+\cos ^{2} \theta_{w}\right)\right], \\
& A_{\mu}(\omega)=\frac{G_{F} n_{\gamma}}{\sqrt{2}}\left[-\mathcal{L}_{\mu}+\frac{7 \pi^{4}}{60 \zeta(3)} \frac{\omega T}{M_{W}^{2}} \cos ^{2} \theta_{w}\right], \\
& B_{e}(k)=-\frac{G_{F} n_{\gamma}}{\sqrt{2}} \frac{7 \pi^{4}}{180 \zeta(3)} \frac{k T}{M_{W}^{2}}\left(2+\cos ^{2} \theta_{w}\right), \\
& B_{\mu}(k)=-\frac{G_{F} n_{\gamma}}{\sqrt{2}} \frac{7 \pi^{4}}{180 \zeta(3)} \frac{k T}{M_{W}^{2}} \cos ^{2} \theta_{w} .
\end{aligned}
$$

where

$$
\begin{aligned}
-\mathcal{L}_{e} & =-\frac{1}{2} L_{\nu_{e}}+L_{\nu_{\mu}}-3 L_{e}-L_{n} \\
-\mathcal{L}_{\mu} & =-\frac{1}{2} L_{\nu_{\mu}}+L_{\nu_{e}}-L_{n}
\end{aligned}
$$

Where in the temperature regime $m_{e} \ll T \ll m_{\mu}$ we consistently neglected the muon contribution to the non-local terms proportional to $\omega, k$ in the exchange diagrams.

The above expressions also reveal the importance of the temperature region $T \gtrsim 5 \mathrm{MeV}$. Assuming that all asymmetries are of the same order as the baryon asymmetry, namely $L_{i} \sim 10^{-9}$, we see that for $\omega \sim k \sim T$ the factor $T^{2} / M_{W}^{2} \gg 10^{-9}$ for $T \gtrsim 5 \mathrm{MeV}$.

We will discuss below that in this region there is also a resonance in the mixing angle in agreement with the results in $[30]$. 


\section{2. $\mathbf{m}_{\mathbf{e}}, \mathbf{m}_{\mu} \ll \mathbf{T} \ll \mathbf{M}_{\mathbf{W}}$}

This temperature region is important because the non-local contributions are much larger than that of the lepton and neutrino asymmetries, assuming both to be of the same order $\sim 10^{-9}$ and are the same for both leptons if their masses are neglected. Therefore, if the contribution from the lepton and neutrino asymmetries is neglected, and terms of $\mathcal{O}\left(m_{e}^{2} / T^{2}\right) ; \mathcal{O}\left(m_{\mu}^{2} / T^{2}\right)$ are neglected, the matrices $\mathbb{A}, \mathbb{B}$ become proportional to the identity. In this case the mixing angle would be the same as in the vacuum. We will see however, that keeping terms of $\mathcal{O}\left(m_{e}^{2} / T^{2}\right) ; \mathcal{O}\left(m_{\mu}^{2} / T^{2}\right)$ leads to a very different result, namely the vanishing of the mixing angle for negative helicity neutrinos or positive helicity antineutrinos in this temperature range.

For $T \gg m_{\mu} \sim 100 \mathrm{MeV}$ the temperature is larger than the critical temperature for deconfinement in QCD $T_{c} \sim 160 \mathrm{MeV}$. Therefore, the medium contains free quarks but no nucleons. Since both $u$ and $d$ quarks have masses smaller than $10 \mathrm{MeV}$, their masses can be neglected. We only include in our description the two lightest quark degrees of freedom consistently with keeping only a weak doublet. We also assume that there is vanishing strangeness in the medium.

As a result, the corresponding $A_{e, \mu}(\omega), B_{e, \mu}(k)$ are now given by

$$
\begin{aligned}
A_{e}(\omega)= & -\frac{3 G_{F}}{\sqrt{2}} L_{e} n_{\gamma}+\frac{7 \pi^{2}}{15 \sqrt{2}} \frac{G_{F} \omega T^{4}}{M_{W}^{2}}-\frac{3 G_{F}}{2 \sqrt{2}} L_{\nu_{e}} n_{\gamma}+\frac{7 \pi^{2}}{30 \sqrt{2}} \frac{G_{F} \omega T^{4}}{M_{Z}^{2}} \\
& +\frac{G_{F}}{\sqrt{2}} L_{\nu_{e}} n_{\gamma}+\frac{G_{F}}{\sqrt{2}} L_{\nu_{\mu}} n_{\gamma} \\
& +\frac{2 G_{F}}{\sqrt{2}}\left(-\frac{1}{2}+2 \sin ^{2} \theta_{w}\right) L_{e} n_{\gamma}+\frac{2 G_{F}}{\sqrt{2}}\left(-\frac{1}{2}+2 \sin ^{2} \theta_{w}\right) L_{\mu} n_{\gamma} \\
& +\frac{6 G_{F}}{\sqrt{2}}\left(\frac{1}{2}-\frac{4}{3} \sin ^{2} \theta_{w}\right) L_{u} n_{\gamma}+\frac{6 G_{F}}{\sqrt{2}}\left(-\frac{1}{2}+\frac{2}{3} \sin ^{2} \theta_{w}\right) L_{d} n_{\gamma}, \\
A_{\mu}(\omega)= & -\frac{3 G_{F}}{\sqrt{2}} L_{\mu} n_{\gamma}+\frac{7 \pi^{2}}{15 \sqrt{2}} \frac{G_{F} \omega T^{4}}{M_{W}^{2}}-\frac{3 G_{F}}{2 \sqrt{2}} L_{\nu_{\mu}} n_{\gamma}+\frac{7 \pi^{2}}{30 \sqrt{2}} \frac{G_{F} \omega T^{4}}{M_{Z}^{2}} \\
& +\frac{G_{F}}{\sqrt{2}} L_{\nu_{e}} n_{\gamma}+\frac{G_{F}}{\sqrt{2}} L_{\nu_{\mu}} n_{\gamma} \\
& +\frac{2 G_{F}}{\sqrt{2}}\left(-\frac{1}{2}+2 \sin ^{2} \theta_{w}\right) L_{e} n_{\gamma}+\frac{2 G_{F}}{\sqrt{2}}\left(-\frac{1}{2}+2 \sin ^{2} \theta_{w}\right) L_{\mu} n_{\gamma} \\
& +\frac{6 G_{F}}{\sqrt{2}}\left(\frac{1}{2}-\frac{4}{3} \sin ^{2} \theta_{w}\right) L_{u} n_{\gamma}+\frac{6 G_{F}}{\sqrt{2}}\left(-\frac{1}{2}+\frac{2}{3} \sin ^{2} \theta_{w}\right) L_{d} n_{\gamma}, \\
B_{e}(k)= & -\frac{7 \pi^{2}}{45 \sqrt{2}} \frac{G_{F} k T^{4}}{M_{W}^{2}}\left[1-\frac{30}{7 \pi^{2}}\left(\frac{m_{e}}{T}\right)^{2}\right]-\frac{7 \pi^{2}}{90 \sqrt{2}} \frac{G_{F} k T^{4}}{M_{Z}^{2}}, \\
B_{\mu}(k)= & -\frac{7 \pi^{2}}{45 \sqrt{2}} \frac{G_{F}}{M_{W}^{2}}\left[1-\frac{30}{7 \pi^{2}}\left(\frac{m_{\mu}}{T}\right)^{2}\right]-\frac{7 \pi^{2}}{90 \sqrt{2}} \frac{G_{F} k T^{4}}{M_{Z}^{2}} .
\end{aligned}
$$

Charge neutrality of the medium leads to the constraint $4 L_{u}-L_{d}-3 L_{e}=0$, which leads to the following simplified expressions:

$$
\begin{aligned}
& A_{e}(\omega)=\frac{G_{F} n_{\gamma}}{\sqrt{2}}\left[\widetilde{-\mathcal{L}_{e}}+\frac{7 \pi^{4}}{60 \zeta(3)} \frac{\omega T}{M_{W}^{2}}\left(2+\cos ^{2} \theta_{w}\right)\right], \\
& A_{\mu}(\omega)=\frac{G_{F} n_{\gamma}}{\sqrt{2}}\left[-\widetilde{\mathcal{L}_{\mu}}+\frac{7 \pi^{4}}{60 \zeta(3)} \frac{\omega T}{M_{W}^{2}}\left(2+\cos ^{2} \theta_{w}\right)\right], \\
& B_{e}(k)=-\frac{G_{F} n_{\gamma}}{\sqrt{2}} \frac{7 \pi^{4}}{180 \zeta(3)} \frac{k T}{M_{W}^{2}}\left[2+\cos ^{2} \theta_{w}-\frac{60}{7 \pi^{2}}\left(\frac{m_{e}}{T}\right)^{2}\right], \\
& B_{\mu}(k)=-\frac{G_{F} n_{\gamma}}{\sqrt{2}} \frac{7 \pi^{4}}{180 \zeta(3)} \frac{k T}{M_{W}^{2}}\left[2+\cos ^{2} \theta_{w}-\frac{60}{7 \pi^{2}}\left(\frac{m_{\mu}}{T}\right)^{2}\right],
\end{aligned}
$$

where

$$
\begin{aligned}
-\widetilde{\mathcal{L}_{e}} & =-\frac{1}{2} L_{\nu_{e}}+L_{\nu_{\mu}}-3 L_{e}+\left(1-4 \sin ^{2} \theta_{w}\right)\left(2 L_{e}-L_{\mu}\right)-\left(1-8 \sin ^{2} \theta_{w}\right) L_{u}-2 L_{d} \\
-\widetilde{\mathcal{L}_{\mu}} & =-\frac{1}{2} L_{\nu_{\mu}}+L_{\nu_{e}}-3 L_{\mu}+\left(1-4 \sin ^{2} \theta_{w}\right)\left(2 L_{e}-L_{\mu}\right)-\left(1-8 \sin ^{2} \theta_{w}\right) L_{u}-2 L_{d} .
\end{aligned}
$$


In the limit when $T \gg m_{e, \mu}$ both leptons become ultrarelativistic and a CP-symmetric medium becomes flavor blind to the weak interactions. In this case we must keep terms of $\mathcal{O}\left(m_{e, \mu} / T\right)$ to understand the nature of oscillations and mixing.

\section{DISPERSION RELATIONS, MIXING ANGLES AND RESONANCES IN THE MEDIUM}

The neutrino dispersion relations and mixing angles in the medium are obtained by diagonalizing the homogeneous effective Dirac equation in the medium, namely by setting $\eta(\omega, k)=0$ in eq.(II.19). Using the results obtained above, the homogeneous Dirac equation in frequency and momentum becomes

$$
\left[\gamma^{0} \omega \mathbb{1}-\vec{\gamma} \cdot \widehat{\mathbf{k}} k \mathbb{1}-\mathbb{M}+\left(\gamma^{0} \mathbb{A}(\omega)-\vec{\gamma} \cdot \widehat{\mathbf{k}} \mathbb{B}(k)\right) L\right] \psi(\omega, k)=0
$$

where $\mathbb{1}$ is the $2 \times 2$ identity matrix in the flavor basis in which the field $\psi(\omega, k)$ is given by

$$
\psi(\omega, k)=\left(\begin{array}{c}
\nu_{e}(\omega, k) \\
\nu_{\mu}(\omega, k)
\end{array}\right),
$$

with $\nu_{e}(\omega, k)$ and $\nu_{\mu}(\omega, k)$ each being a 4 -component Dirac spinor.

If we multiply the effective Dirac equation (IV.1) by the chiral projectors $R$ and $L$ respectively from the left, we obtain

$$
\begin{aligned}
& \left(\gamma^{0} \mathbb{W}-\vec{\gamma} \cdot \widehat{\mathbf{k}} \mathbb{K}\right) \psi_{L}-\mathrm{IM} \psi_{R}=0, \\
& \left(\gamma^{0} \omega \mathbb{1}-\vec{\gamma} \cdot \widehat{\mathbf{k}} k \mathbb{1}\right) \psi_{R}-\mathrm{IM} \psi_{L}=0,
\end{aligned}
$$

where we have defined the flavor matrices

$$
\mathbb{W}=\omega \mathbb{1}+\mathbb{A} \quad, \quad \mathbb{K}=k \mathbb{1}+\mathbb{B} .
$$

The set of equations (IV.3) and (IV.4) couple $\psi_{L}$ and $\psi_{R}$ together. To solve the equations, we first multiply (IV.3) by $\left(\gamma^{0} \omega-\vec{\gamma} \cdot \widehat{\mathbf{k}} k\right) \mathbb{1}$ from the left and use eq.(IV.4) to obtain an equation for $\psi_{L}$ which can be written in terms of the helicity operator $\hat{h}(\widehat{\mathbf{k}})=\gamma^{0} \vec{\gamma} \cdot \widehat{\mathbf{k}} \gamma^{5}$, as follows

$$
\left[\omega \mathbb{W}-k \mathbb{K}+\hat{h}(\widehat{\mathbf{k}})(\omega \mathbb{K}-\mathbb{W} k)-\mathbb{M}^{2}\right] \psi_{L}=0,
$$

and the right handed component is given by

$$
\psi_{R}(\omega, k)=\mathbb{M} \gamma^{0} \frac{[\omega+\hat{h}(\widehat{\mathbf{k}}) k]}{\omega^{2}-k^{2}} \psi_{L}(\omega, k) .
$$

It is convenient to separate the Dirac and flavor structure to simplify the study. This is achieved most economically in the chiral representation of the Dirac matrices, in which

$$
\begin{gathered}
\gamma^{0}=\left(\begin{array}{cc}
0 & -\mathbb{1} \\
-\mathbb{1} & 0
\end{array}\right) ; \gamma^{5}=\left(\begin{array}{cc}
\mathbb{1} & 0 \\
0 & -\mathbb{1}
\end{array}\right), \\
\vec{\gamma} \cdot \widehat{\mathbf{k}}=\left(\begin{array}{cc}
0 & \vec{\sigma} \cdot \widehat{\mathbf{k}} \mathbb{1} \\
-\vec{\sigma} \cdot \widehat{\mathbf{k}} \mathbb{1} & 0
\end{array}\right) ; \hat{h}(k)=\vec{\sigma} \cdot \widehat{\mathbf{k}}\left(\begin{array}{ll}
\mathbb{1} & 0 \\
0 & \mathbb{1}
\end{array}\right),
\end{gathered}
$$

and by introducing the two component Weyl spinors $v^{(h)}(\widehat{\mathbf{k}})$ eigenstates of helicity,

$$
\vec{\sigma} \cdot \widehat{\mathbf{k}} v^{(h)}(\widehat{\mathbf{k}})=h v^{(h)}(\widehat{\mathbf{k}}) ; h= \pm 1 .
$$

These spinors are normalized so that

$$
\left(v^{(h)}(\widehat{\mathbf{k}})\right)^{\dagger} v^{\left(h^{\prime}\right)}(\widehat{\mathbf{k}})=\delta_{h, h^{\prime}}
$$


In terms of these helicity eigenstates, a general flavor doublet of left (L) and right (R) handed Dirac spinors can be written

$$
\psi_{L}=\sum_{h= \pm 1}\left(\begin{array}{c}
0 \\
v^{(h)} \otimes \varphi^{(h)}
\end{array}\right),
$$

and

$$
\psi_{R}=\sum_{h= \pm 1}\left(\begin{array}{c}
v^{(h)} \otimes \xi^{(h)} \\
0
\end{array}\right)
$$

where $\varphi^{(h)} ; \xi^{(h)}$ are flavor doublets. We have purposely left the arguments unspecified because this expansion will be used in real time as well as for the Fourier and Laplace transforms respectively. We need both positive and negative helicity eigenstates because the four independent degrees of freedom for each flavor are positive and negative energy and positive and negative helicity.

Projecting eq. (IV.6) onto the helicity eigenstates $v^{(h)}(\widehat{\mathbf{k}})$ we obtain an equation for the flavor doublet $\varphi^{(h)}(\omega, k)$, namely

$$
\left[\left(\omega^{2}-k^{2}\right) \mathbb{1}+(\omega-h k)(\mathbb{A}+h \mathbb{B})-\mathbb{M}^{2}\right] \varphi^{(h)}(\omega, k)=0 .
$$

Projecting eq. (IV.7) onto helicity eigenstates yields the relation

$$
\xi^{(h)}(\omega, k)=-\mathbb{M} \frac{(\omega+h k)}{\omega^{2}-k^{2}} \varphi^{(h)}(\omega, k) .
$$

Writing the doublet $\varphi^{(h)}(\omega, k)$ in the flavor basis as

$$
\varphi^{(h)}(\omega, k)=\left(\begin{array}{c}
\nu_{e}^{(h)}(\omega, k) \\
\nu_{\mu}^{(h)}(\omega, k)
\end{array}\right),
$$

leads to the following matrix form for Eq. (IV.14)

$$
\left(\begin{array}{cc}
a^{(h)} & b \\
b & c^{(h)}
\end{array}\right)\left(\begin{array}{c}
\nu_{e}^{(h)} \\
\nu_{\mu}^{(h)}
\end{array}\right)=0
$$

where the matrix elements in the flavor basis are given by

$$
\begin{aligned}
a^{(h)} & =\omega^{2}-k^{2}+(\omega-h k)\left(A_{e}+h B_{e}\right)-\frac{1}{2}\left(M_{1}^{2}+M_{2}^{2}\right)-\frac{1}{2}\left(M_{1}^{2}-M_{2}^{2}\right) \cos (2 \theta), \\
b & =\frac{1}{2}\left(M_{1}^{2}-M_{2}^{2}\right) \sin (2 \theta), \\
c^{(h)} & =\omega^{2}-k^{2}+(\omega-h k)\left(A_{\mu}+h B_{\mu}\right)-\frac{1}{2}\left(M_{1}^{2}+M_{2}^{2}\right)+\frac{1}{2}\left(M_{1}^{2}-M_{2}^{2}\right) \cos (2 \theta) .
\end{aligned}
$$

Let us introduce a doublet of mass eigenstates in the medium

$$
\chi^{(h)}(\omega, k)=\left(\begin{array}{c}
\nu_{1}(\omega, k) \\
\nu_{2}(\omega, k)
\end{array}\right)
$$

related to the flavor doublet $\varphi^{(h)}(\omega, k)$ by a unitary transformation $U_{m}^{(h)}$ with

$$
\begin{gathered}
U_{m}^{(h)}=\left(\begin{array}{cc}
\cos \theta_{m}^{(h)} & \sin \theta_{m}^{(h)} \\
-\sin \theta_{m}^{(h)} & \cos \theta_{m}^{(h)}
\end{array}\right), \\
\varphi^{(h)}(\omega, k)=U_{m}^{(h)} \chi^{(h)}(\omega, k) ; \quad \xi^{(h)}(\omega, k)=U_{m}^{(h)} \zeta^{(h)}(\omega, k) .
\end{gathered}
$$


The mixing angle in the medium for states with helicity $h, \theta_{m}^{(h)}$ is obtained by requiring that the unitary transformation eq.(IV.22) diagonalizes the matrix equation eq.(IV.17). The eigenvalue equation in diagonal form is given by

$$
\left\{\omega^{2}-k^{2}+\frac{1}{2} S_{h}(\omega, k)-\frac{1}{2}\left(M_{1}^{2}+M_{2}^{2}\right)-\frac{1}{2} \delta M^{2}\left[\left(\cos 2 \theta-\frac{\Delta_{h}(\omega, k)}{\delta M^{2}}\right)^{2}+\sin ^{2} 2 \theta\right]^{\frac{1}{2}}\left(\begin{array}{cc}
1 & 0 \\
0 & -1
\end{array}\right)\right\} \chi^{(h)}(\omega, k)=0,
$$

where $S_{h}(\omega, k), \delta M^{2}$ and $\Delta_{h}$ are respectively given by

$$
\begin{aligned}
S_{h}(\omega, k) & =(\omega-h k)\left[A_{e}(\omega)+A_{\mu}(\omega)+h B_{e}(k)+h B_{\mu}(k)\right] \\
\delta M^{2} & =M_{1}^{2}-M_{2}^{2}, \\
\Delta_{h}(\omega, k) & =(\omega-h k)\left[A_{e}(\omega)-A_{\mu}(\omega)+h B_{e}(k)-h B_{\mu}(k)\right] .
\end{aligned}
$$

The mixing angle in the medium is determined by the relation

$$
\tan \left[2 \theta_{m}^{(h)}\right]=\frac{2 b}{c^{(h)}-a^{(h)}}=\frac{\delta M^{2} \sin (2 \theta)}{\delta M^{2} \cos (2 \theta)-\Delta_{h}(\omega, k)},
$$

or alternatively by the more familiar relation

$$
\sin 2 \theta_{m}^{(h)}=\frac{\sin 2 \theta}{\left[\left(\cos 2 \theta-\frac{\Delta_{h}(\omega, k)}{\delta M^{2}}\right)^{2}+\sin ^{2} 2 \theta\right]^{\frac{1}{2}}}
$$

We note that the neutrino mass eigenvalues as well as the mixing angle depends on $k$ as well as on the helicity eigenvalue $h$. This is one of the novel results which has not been obtained before simply because only left handed negative helicity neutrinos were considered in the literature 1, 11, 12, 13, 14, 15.

The right handed components are obtained from the left handed ones by performing the unitary transformation eq. (IV.22) on eq.(IV.15). The relation (IV.15) leads to the following expressions

$$
\zeta^{(h)}(\omega, k)=-\frac{\omega+h k}{\omega^{2}-k^{2}} \bar{M}\left[\mathbb{1}+\frac{\delta M^{2}}{4 \bar{M}^{2}}\left(\begin{array}{cc}
\bar{C} & \bar{S} \\
\bar{S} & -\bar{C}
\end{array}\right)\right] \chi^{(h)}(\omega, k),
$$

where $\bar{M}=\frac{1}{2}\left(M_{1}+M_{2}\right)$ and

$$
\bar{C}=\cos \left[2 \theta_{m}^{(h)}-2 \theta\right] \quad, \quad \bar{S}=\sin \left[2 \theta_{m}^{(h)}-2 \theta\right] .
$$

\section{A. Eigenvectors and dispersion relations}

Eq.(IV.24) has the following eigenvectors in the basis of mass eigenstates:

$$
\begin{aligned}
& \chi_{1}^{(h)}(\omega, k)=\nu_{1}^{(h)}(\omega, k)\left(\begin{array}{l}
1 \\
0
\end{array}\right), \\
& \zeta_{1}^{(h)}(\omega, k)=-\nu_{1}^{(h)}(\omega, k) \frac{\omega+h k}{\omega^{2}-k^{2}} \bar{M}\left[\left(\begin{array}{l}
1 \\
0
\end{array}\right)+\frac{\delta M^{2}}{4 \bar{M}^{2}}\left(\begin{array}{c}
\bar{C} \\
\bar{S}
\end{array}\right)\right],
\end{aligned}
$$

and

$$
\begin{aligned}
\chi_{2}^{(h)}(\omega, k) & =\nu_{2}^{(h)}(\omega, k)\left(\begin{array}{l}
0 \\
1
\end{array}\right) \\
\zeta_{2}^{(h)}(\omega, k) & =-\nu_{2}^{(h)}(\omega, k) \frac{\omega+h k}{\omega^{2}-k^{2}} \bar{M}\left[\left(\begin{array}{l}
0 \\
1
\end{array}\right)+\frac{\delta M^{2}}{4 \bar{M}^{2}}\left(\begin{array}{c}
\bar{S} \\
-\bar{C}
\end{array}\right)\right]
\end{aligned}
$$

The corresponding doublets in the flavor basis can be obtained by the unitary transformation eq. (IV.22). 
The eigenvalues are found in perturbation theory consistently up to $\mathcal{O}\left(G_{F}\right)$ by writing

$$
\omega_{a}^{(h)}(k, \pm)= \pm\left[E_{a}(k)+\delta \omega_{a}^{(h)}(k, \pm)\right] \quad, \quad a=1,2
$$

with

$$
E_{1,2}(k)=\sqrt{k^{2}+M_{1,2}^{2}}
$$

We find,

$$
\begin{aligned}
& \delta \omega_{1}^{(h)}(k, \pm)=-\frac{1}{4 E_{1}(k)}\left\{S_{h}\left( \pm E_{1}(k), k\right)-\delta M^{2}\left[\left[\left(\cos 2 \theta-\frac{\Delta_{h}\left( \pm E_{1}(k), k\right)}{\delta M^{2}}\right)^{2}+\sin ^{2} 2 \theta\right]^{\frac{1}{2}}-1\right]\right\} \\
& \left.\delta \omega_{2}^{(h)}(k, \pm)=-\frac{1}{4 E_{2}(k)}\left\{S_{h}\left( \pm E_{2}(k), k\right)+\delta M^{2}\left[\left(\cos 2 \theta-\frac{\Delta_{h}\left( \pm E_{2}(k), k\right)}{\delta M^{2}}\right)^{2}+\sin ^{2} 2 \theta\right]-1\right]\right\}
\end{aligned}
$$

It is important to highlight that whereas the mixing angle only depends on $\Delta_{h}$, the medium corrections to the frequencies also depend on $S_{h}$. This is important because even when in the case when the matrices $\mathbb{A}$, B become proportional to the identity, in which case $\Delta_{h}=0$ and the mixing angle in the medium coincides with that of the vacuum, the frequencies and in particular the oscillation frequency still receives medium corrections.

\section{B. Resonances}

The condition for resonant oscillations is that the mixing $\tan \left[2 \theta_{m}^{(h)}\right]$ reaches a maximum (infinity) as a function of a parameter, temperature, density or energy. From eq. (IV.28) a resonance takes place when

$$
\frac{\Delta_{h}(\omega, k)}{\delta M^{2}}=\cos 2 \theta
$$

where $\omega=\omega_{a}^{(h)}(k, \pm)$ correspond to the dispersion relations for the propagating modes in the medium, given by eq.(IV.36). To leading order in $G_{F}$ the in-medium dispersion relation can be approximated by the free field dispersion relation $\omega_{a}^{(h)}(k, \pm) \approx \pm \sqrt{k^{2}+M_{1,2}^{2}}$. The relativistic limit is warranted because the neutrino momenta in the plasma is $k \gg M_{1,2} \sim \mathrm{eV}$. Furthermore, under the assumption that the hierarchy of vacuum mass eigenstates is nearly degenerate, namely $\left|\delta M^{2} / \bar{M}^{2}\right| \ll 1$, as seems to be supported by the experimental data, the dispersion relations can be further approximated as follows

$$
\omega_{a}^{(h)}(k, \pm) \approx \lambda k\left(1+\frac{\bar{M}^{2}}{2 k^{2}}\right) \quad ; \quad \lambda= \pm 1
$$

It is convenient to introduce the following notation

$$
\begin{aligned}
\mathcal{L}_{9} & =10^{9}\left(\mathcal{L}_{e}-\mathcal{L}_{\mu}\right) \\
\delta_{5} & =10^{5}\left(\frac{\delta M^{2}}{\mathrm{eV}^{2}}\right)
\end{aligned}
$$

If the lepton and neutrino asymmetries are of the same order of the baryon asymmetry, then $0.2 \lesssim\left|\mathcal{L}_{9}\right| \lesssim 0.7$ and the fitting from solar and KamLAND data suggests $\left|\delta_{5}\right| \approx 8$. Using the approximations leading to eq. (IV.41) the ratio $\Delta_{h} / \delta M^{2}$ can be written compactly from eq.(IV.27).

We study separately the cases $m_{e} \ll T \ll m_{\mu}$ and $m_{e}, m_{\mu} \ll T \ll M_{W}$. 


$$
\text { 1. } \mathbf{m}_{\mathbf{e}} \ll \mathbf{T} \ll \mathbf{m}_{\mu}
$$

- case I: $\omega=k+\frac{\bar{M}^{2}}{2 k}, h=-1$, positive energy, negative helicity neutrinos:

$$
\frac{\Delta_{h}}{\delta M^{2}} \approx \frac{4}{\delta_{5}}\left(\frac{0.1 T}{\mathrm{MeV}}\right)^{4} \frac{k}{T}\left[-\mathcal{L}_{9}+\left(\frac{2 T}{\mathrm{MeV}}\right)^{2} \frac{k}{T}\right] .
$$

Where we have neglected $\frac{\bar{M}^{2}}{k^{2}}$. For fixed temperature, the resonance condition eq. (IV.40) is fulfilled for the value of neutrino momentum given by

$$
k=\frac{(\mathrm{MeV})^{2}}{8 T}\left\{\mathcal{L}_{9}+\left[\mathcal{L}_{9}^{2}+16 \delta_{5} \cos 2 \theta\left(\frac{200 \mathrm{MeV}}{T}\right)^{2}\right]^{\frac{1}{2}}\right\} .
$$

Hence, for $\delta_{5} \cos 2 \theta>0$, there is always a resonance. If $\left|\mathcal{L}_{9}\right| \lesssim 1$, then for neutrino momenta such that $\sqrt{T k}>1 \mathrm{MeV}$ the non-local term dominates over the asymmetry and the resonance occurs for $k \sim$ $25 \sqrt{\delta_{5} \cos 2 \theta}(\mathrm{MeV})^{3} / T^{2}$. For example, if $T \sim 10 \mathrm{MeV}$, the resonance occurs for $k \sim 1 \mathrm{MeV}$. If $\delta_{5} \cos 2 \theta<0$ there can also be a resonance provided

$$
\frac{\left|\mathcal{L}_{9}\right| T}{200 \mathrm{MeV}}>4 \sqrt{\left|\delta_{5} \cos 2 \theta\right|}
$$

However this inequality requires a large value of $\left|\mathcal{L}_{9}\right|$, for example for $T \sim 10 \mathrm{MeV}$ it requires that $\left|\mathcal{L}_{9}\right| \gtrsim 140$.

- case II: $\omega=k+\frac{\bar{M}^{2}}{2 k}, h=1$, positive energy, positive helicity neutrinos:

$$
\frac{\Delta_{h}}{\delta M^{2}} \approx \frac{10^{-16}}{\delta_{5}}\left(\frac{T}{\mathrm{MeV}}\right)^{2}\left(\frac{\bar{M}}{\mathrm{eV}}\right)^{2}\left[-\mathcal{L}_{9} \frac{T}{k}+2\left(\frac{T}{\mathrm{MeV}}\right)^{2}\right]
$$

Where we have neglected terms of higher order in $\frac{\bar{M}^{2}}{k^{2}}$. Because $\bar{M} \sim 1 \mathrm{eV}$ and $100 \mathrm{MeV} \gg T \gg 1 \mathrm{MeV}$ a resonance would only be available for $k \sim 10^{-16} \mathrm{MeV}$ which is not a relevant range of momenta for neutrinos in the plasma. Therefore, positive helicity neutrinos mix with the vacuum mixing angle.

- case III: $\omega=-k-\frac{\bar{M}^{2}}{2 k}, h=-1$, positive energy, negative helicity anti-neutrinos:

$$
\frac{\Delta_{h}}{\delta M^{2}} \approx \frac{10^{-16}}{\delta_{5}}\left(\frac{T}{\mathrm{MeV}}\right)^{2}\left(\frac{\bar{M}}{\mathrm{eV}}\right)^{2}\left[\mathcal{L}_{9} \frac{T}{k}+2\left(\frac{T}{\mathrm{MeV}}\right)^{2}\right] .
$$

Again in this expression we have neglected higher order terms in $\frac{\bar{M}^{2}}{k^{2}}$. A conclusion similar to that of case II above holds in this case. No resonance is available for relevant values of neutrino momenta within the temperature range in which these results are valid. For the cases II and III the ratio $\left|\Delta_{h} / \delta M^{2}\right| \ll 1$ for all relevant values of the neutrino momentum within the temperature range in which these results are valid. Therefore, negative helicity antineutrinos mix with the vacuum mixing angle, just as positive helicity neutrinos.

- case IV: $\omega=-k-\frac{\bar{M}^{2}}{2 k}, h=1$, positive energy, positive helicity anti-neutrinos:

$$
\frac{\Delta_{h}}{\delta M^{2}} \approx \frac{4}{\delta_{5}}\left(\frac{0.1 T}{\mathrm{MeV}}\right)^{4} \frac{k}{T}\left[\mathcal{L}_{9}+\left(\frac{2 T}{\mathrm{MeV}}\right)^{2} \frac{k}{T}\right] .
$$

Where we have neglected higher order terms in $\frac{\bar{M}^{2}}{k^{2}}$. The position of the resonance in this case is obtained from that in case I above by the replacement $\mathcal{L}_{9} \rightarrow-\mathcal{L}_{9}$, namely for fixed temperature the resonance condition is fulfilled at the value of $k$ given by

$$
k=\frac{(\mathrm{MeV})^{2}}{8 T}\left\{-\mathcal{L}_{9}+\left[\mathcal{L}_{9}^{2}+16 \delta_{5} \cos 2 \theta\left(\frac{200 \mathrm{MeV}}{T}\right)^{2}\right]^{\frac{1}{2}}\right\} .
$$


Again in the temperature range $1 \mathrm{MeV} \ll T \ll 100 \mathrm{MeV}$ there is a resonance if $\delta_{5} \cos 2 \theta>0$ (assuming that $\left.\left|\delta_{5} \cos 2 \theta\right| \sim 1\right)$.

Just as in case I, if $\left|\mathcal{L}_{9}\right| \lesssim 1$ the non-local term dominates over the asymmetry contribution for $\sqrt{T k} \gtrsim 1 \mathrm{MeV}$ and the resonance occurs for $k \sim 25 \sqrt{\delta_{5} \cos 2 \theta}(\mathrm{MeV})^{3} / T^{2}$.

Cases III and IV reveal an interesting feature: only the asymmetry contribution changes sign between neutrinos and antineutrinos whereas the non-local (in space-time) term remains the same.

Together these expressions confirm that if the lepton and neutrino asymmetries are of the same order as the baryon asymmetry, namely $0.2 \lesssim\left|\mathcal{L}_{9}\right| \lesssim 0.7$, then the non-local terms from the exchange diagrams dominate the self-energy for $T \gtrsim 3-5 \mathrm{MeV}$ unless the neutrino in the plasma has a momentum $k$ such that $\sqrt{k T} \ll 0.5 \mathrm{MeV}$.

In summary, for $m_{e} \ll T \ll m_{\mu}$ resonances occur in cases I and IV when $h \lambda<0$ ( $\lambda$ is the sign of the energy eigenvalue). For $h \lambda>0$ (cases II and III) no resonance is available for neutrino momenta $k \sim T \sim$ few MeV and mixing angle in the medium coincides with the vacuum value.

$$
\text { 2. } \mathbf{m}_{\mathbf{e}}, \mathbf{m}_{\mu} \ll \mathbf{T} \ll \mathbf{M}_{\mathbf{W}}
$$

We use $m_{\mu} \approx 106 \mathrm{MeV}$ and find the following simple expressions

- case $\mathbf{I}: \omega=k+\frac{\bar{M}^{2}}{2 k}, h=-1$, positive energy, negative helicity neutrinos:

$$
\frac{\Delta_{h}}{\delta M^{2}} \approx \frac{0.4 \times 10^{12}}{\delta_{5}}\left(\frac{T}{\mathrm{GeV}}\right)^{4} \frac{k}{T}\left[4.83 \frac{k}{T}+10^{-3} \mathcal{L}_{9}\right]
$$

In this case no resonance is available but for neutrinos with extremely low energy and not relevant for the plasma. For example for $T \sim \mathrm{GeV}$ only neutrinos with energy of a few $\mathrm{eV}$ would be potentially resonant, but this momentum range is not a relevant one for neutrinos in the plasma. For neutrinos with energy larger than a few $k e V$ the mixing angle effectively vanishes. Therefore, we conclude that in this temperature regime the mixing angle in the medium for negative helicity neutrinos vanishes.

- case II: $\omega=k+\frac{\bar{M}^{2}}{2 k}, h=1$, positive energy, positive helicity neutrinos:

$$
\frac{\Delta_{h}}{\delta M^{2}} \approx-\frac{10^{-7}}{\delta_{5}}\left(\frac{T}{\mathrm{GeV}}\right)^{2} \frac{T}{k}\left(\frac{\bar{M}}{\mathrm{eV}}\right)^{2}\left[4.83 \frac{k}{T}-10^{-3} \mathcal{L}_{9}\right]
$$

It is clear that for the relevant regime of neutrino momenta in the plasma $\left|\Delta_{h} / \delta M^{2}\right| \ll 1$. Hence the mixing angle in the medium coincides with the vacuum mixing angle. Thus the conclusion in this case is similar to that in the case described by eq. (IV.47), namely positive helicity neutrinos undergo oscillations in the medium with the vacuum mixing angle.

- case III: $\omega=-k-\frac{\bar{M}^{2}}{2 k}, h=-1$, positive energy, negative helicity anti-neutrinos:

$$
\frac{\Delta_{h}}{\delta M^{2}} \approx-\frac{10^{-7}}{\delta_{5}}\left(\frac{T}{\mathrm{GeV}}\right)^{2} \frac{T}{k}\left(\frac{\bar{M}}{\mathrm{eV}}\right)^{2}\left[4.83 \frac{k}{T}+10^{-3} \mathcal{L}_{9}\right]
$$

The result in this case is similar to that of case II above, negative helicity antineutrinos oscillate in the medium with the vacuum mixing angle.

- case IV: $\omega=-k-\frac{\bar{M}^{2}}{2 k}, h=1$, positive energy, positive helicity anti-neutrinos:

$$
\frac{\Delta_{h}}{\delta M^{2}} \approx \frac{0.4 \times 10^{12}}{\delta_{5}}\left(\frac{T}{\mathrm{GeV}}\right)^{4} \frac{k}{T}\left[4.83 \frac{k}{T}-10^{-3} \mathcal{L}_{9}\right]
$$

The conclusion in this case is similar to that of the case described by eq. (IV.51) above, the mixing angle effectively vanishes and oscillations of positive helicity antineutrinos are suppressed in the medium in this temperature range. 
Taken together the above analysis reveals that there is a resonance in the oscillation of negative helicity neutrinos and positive helicity antineutrinos (that is $h \lambda<0$ ) in the temperature range $m_{e} \ll T \ll m_{\mu}$ with a typical neutrino momentum $k \sim T \sim$ few MeV. For $m_{e}, m_{\mu} \ll T \ll M_{W}$ the mixing angle for negative helicity neutrinos and positive helicity antineutrinos (that is $h \lambda<0$ ) effectively vanishes in the medium, and in both temperature ranges positive helicity neutrinos and negative helicity antineutrinos undergo oscillations in the medium with the vacuum mixing angle. We cannot yet conclude that positive helicity neutrinos and negative helicity antineutrinos are sterile, before studying the corrections to the oscillation frequencies.

\section{Oscillation frequencies and time scales}

The oscillation time scale in the medium is given by

$$
\tau_{m}^{(h)}(k, \lambda)=\frac{1}{\left|\omega_{1}^{(h)}(k, \lambda)-\omega_{2}^{(h)}(k, \lambda)\right|}=\frac{1}{\left|E_{1}(k)-E_{2}(k)+\delta \omega_{1}^{(h)}(k, \lambda)-\delta \omega_{2}^{(h)}(k, \lambda)\right|},
$$

where $E_{1,2}(k)=\sqrt{k^{2}+M_{1,2}^{2}}$ and $\lambda=+1$ and $\lambda=-1$ correspond to neutrino and antineutrinos respectively.

The vacuum oscillation time scale is

$$
\tau_{v}(k)=\frac{1}{\left|E_{1}(k)-E_{2}(k)\right|},
$$

therefore, in order to understand the loop corrections to the oscillations time scales, it is convenient to study the ratio

$$
\frac{\tau_{v}(k)}{\tau_{m}^{(h)}(k, \lambda)}=\left|1+\frac{\delta \omega_{1}^{(h)}(k, \lambda)-\delta \omega_{2}^{(h)}(k, \lambda)}{E_{1}(k)-E_{2}(k)}\right| .
$$

Typical neutrino momenta in the plasma are ultrarelativistic, hence we approximate

$$
E_{1}(k)-E_{2}(k) \approx \frac{\delta M^{2}}{2 k}=\frac{\delta_{5}}{2} \frac{10^{-11} \mathrm{eV}}{(k / \mathrm{MeV})} .
$$

furthermore to leading order in $G_{F}$ we replace $\omega_{a}^{(h)}(k, \lambda) \approx \lambda k$ in the arguments of $A_{e, \mu}(\omega)$.

The term $\delta \omega_{1}^{(h)}(k, \lambda)-\delta \omega_{2}^{(h)}(k, \lambda)$ represents the correction to the vacuum oscillation time scale due to the medium effect. While the general form of these corrections are cumbersome, we can extract simplified expressions in three relevant limits.

- I Resonant case: $\frac{\Delta_{h}\left(\lambda E_{1,2}, k\right)}{\delta M^{2}} \approx \cos 2 \theta:$

In section IVB above we found that resonant flavor oscillations can occur only for $\lambda=+1, h=-1$ and $\lambda=-1, h=+1$. In both these cases we obtain,

$$
\begin{aligned}
\delta \omega_{1}^{(h)}(k, \lambda)-\delta \omega_{2}^{(h)}(k, \lambda)= & -\frac{\delta M^{2}}{8 k^{2}}\left[A_{e}(k)+A_{\mu}(k)+B_{e}(k)+B_{\mu}(k)\right] \\
& -\frac{\delta M^{2}}{2 k^{2}}\left(1+\frac{\bar{M}^{2}}{2 k^{2}}\right) k(1-\sin 2 \theta)
\end{aligned}
$$

- II vanishing mixing angle : $\left|\frac{\Delta_{h}\left(\lambda E_{1,2}, k\right)}{\delta M^{2}}\right| \gg 1$ :

In this limit, $\theta_{m}^{(h)} \sim 0$ and neutrino flavor mixing is suppressed. In section IVB above, we found that this occurs only for $\lambda=+1, h=-1$ or $\lambda=-1, h=+1$. Furthermore, $\Delta_{h}\left(\lambda E_{1,2}, k\right)$ is always positive definite in both temperature limits considered here $m_{e} \ll T \ll m_{\mu}$ and $m_{e}, m_{\mu} \ll T \ll M_{W}$. 
In this case we obtain

$$
\begin{aligned}
\delta \omega_{1}^{(h)}(k, \lambda)-\delta \omega_{2}^{(h)}(k, \lambda)= & -\frac{\delta M^{2}}{8 k^{2}}\left[A_{e}(k)+A_{\mu}(k)+B_{e}(k)+B_{\mu}(k)\right] \\
& +\operatorname{sign}\left(\delta M^{2}\right)\left[A_{e}(k)-A_{\mu}(k)-B_{e}(k)+B_{\mu}(k)\right] .
\end{aligned}
$$

- III vacuum mixing: $\left|\frac{\Delta_{h}\left(\lambda E_{1,2}, k\right)}{\delta M^{2}}\right| \ll 1$ :

In this limit $\theta_{m}^{(h)} \approx \theta$. In section IVB above we found that this case occurs for $\lambda=+1, h=+1$ or $\lambda=-1, h=-1$. In both these cases we find

$$
\begin{aligned}
\delta \omega_{1}^{(h)}(k, \lambda)-\delta \omega_{2}^{(h)}(k, \lambda)= & -\frac{\delta M^{2}}{8 k^{2}}\left[A_{e}(k)+A_{\mu}(k)+B_{e}(k)+B_{\mu}(k)\right] \\
& -\frac{\bar{M}^{2}}{4 k^{2}} \cos 2 \theta\left[A_{e}(k)-A_{\mu}(k)+B_{e}(k)-B_{\mu}(k)\right] .
\end{aligned}
$$

We now study these simplified expressions in the different regimes of temperature and for the different helicities components. The most relevant cosmological regime corresponds to momenta of the order of the temperature, hence we will focus on the regime in which the non-local (in space-time) contributions from the exchange diagrams dominate over the lepton-neutrino asymmetries. Taken together these simplifications allow us to study the relevant cosmological range of neutrino energies in a clear manner.

$$
\text { 1. } \mathbf{m}_{\mathbf{e}} \ll \mathbf{T} \ll \mathbf{m}_{\mu}
$$

- case $\mathbf{I}: \omega_{1,2}(k, \lambda)=\lambda\left(k+\frac{M_{1,2}^{2}}{2 k}\right) ; \lambda=+1, h=-1$ and $\lambda=-1, h=+1$ :

As observed in section (IVB) the non-local terms become dominant for $\sqrt{T k} \geq 1 \mathrm{MeV}$ which is of course consistent with the ultrarelativistic limit $\bar{M}^{2} / 2 k^{2} \ll 1$. Furthermore in the temperature range of interest in this study, the factors $A_{e, \mu}(k)$ and $B_{e, \mu}(k)$ are of the order $G_{F} k T^{4} / M_{W}^{2} \sim 10^{-9}(T / \mathrm{GeV})^{4} k \ll k$. Therefore, near the resonance which occurs when $T^{2} k \sim 25 \sqrt{\delta_{5} \cos 2 \theta} \mathrm{MeV}^{3}$, the expression (IV.59) simplifies to

$$
\delta \omega_{1}^{(h)}(k, \lambda)-\delta \omega_{2}^{(h)}(k, \lambda) \approx-\frac{\delta M^{2}}{2 k}(1-\sin 2 \theta) .
$$

The ratio of the oscillation time scales (IV.57) becomes

$$
\frac{\tau_{v}(k)}{\tau_{m}^{(h)}(k, \lambda)} \sim|\sin 2 \theta|<1
$$

Therefore, for small vacuum mixing angle there is a considerable slow down of oscillations. Resonant flavor mixing in the medium occurs on longer time scales than in the vacuum.

For large neutrino energy, well outside the resonance region for $T^{2} k \gg 25 \sqrt{\left|\delta_{5} \cos 2 \theta\right|} \mathrm{MeV}^{3}$, eq. (IV.44) indicates that $\left|\Delta_{h}\left(\lambda E_{1,2}, k\right) / \delta M^{2}\right| \gg 1$. In this high energy regime we find that

$$
\begin{aligned}
\delta \omega_{1}^{(h)}(k, \lambda)-\delta \omega_{2}^{(h)}(k, \lambda) & \approx \operatorname{sign}\left(\delta M^{2}\right)\left[A_{e}(k)-A_{\mu}(k)-B_{e}(k)+B_{\mu}(k)\right] \\
& =\operatorname{sign}\left(\delta M^{2}\right) \frac{28 \pi^{2}}{45 \sqrt{2}} \frac{G_{F} k T^{4}}{M_{W}^{2}} \\
& \simeq 7.9 \times 10^{-15} \mathrm{eV} \operatorname{sign}\left(\delta M^{2}\right) \frac{k}{\mathrm{MeV}}\left(\frac{T}{\mathrm{MeV}}\right)^{4}
\end{aligned}
$$


therefore neutrino oscillations are suppressed by a vanishingly small mixing angle and the ratio of time scales (IV.57) becomes

$$
\frac{\tau_{v}(k)}{\tau_{m}^{(h)}(k, \lambda)} \sim\left|1+\frac{10^{-3}}{\left|\delta_{5}\right|}\left(\frac{k T^{2}}{\mathrm{MeV}^{3}}\right)^{2}\right| .
$$

A considerable speed-up of oscillations occurs for $k T^{2} \gtrsim 100 \mathrm{MeV}^{3}$ since then $\tau_{m}^{(h)}(k, \lambda) \ll \tau_{v}(k)$. In this case, off-resonance flavor mixing is suppressed not only by a small mixing angle in the medium but also by a rapid decoherence and dephasing of the oscillations.

- case II: $\omega_{1,2}(k, \lambda)=\lambda\left(k+\frac{M_{1,2}^{2}}{2 k}\right) ; \lambda=+1, h=+1$ and $\lambda=-1, h=-1$ :

The results of section IVB (see eq. (IV.47) indicate that in this case $\left|\Delta_{h}\left(\lambda E_{1,2}, k\right) / \delta M^{2}\right| \ll 1$, corresponding to the mixing angle in the medium being the same as in the vacuum. As a result,

$$
\begin{aligned}
\delta \omega_{1}^{(h)}(k, \lambda)-\delta \omega_{2}^{(h)}(k, \lambda) & \approx-\frac{\bar{M}^{2}}{4 k^{2}} \cos 2 \theta\left[A_{e}(k)-A_{\mu}(k)+B_{e}(k)-B_{\mu}(k)\right] \\
& =-\frac{\bar{M}^{2}}{4 k^{2}} \cos 2 \theta \frac{14 \pi^{2}}{45 \sqrt{2}} \frac{G_{F} k T^{4}}{M_{W}^{2}} \\
& \simeq-6.1 \times 10^{-29} \mathrm{eV} \cos 2 \theta\left(\frac{k}{\mathrm{MeV}}\right)^{-1}\left(\frac{T}{\mathrm{MeV}}\right)^{4} .
\end{aligned}
$$

and the ratio of time scales (IV.57) becomes

$$
\frac{\tau_{v}(k)}{\tau_{m}^{(h)}(k, \lambda)} \simeq\left|1-\frac{10^{-17} \cos 2 \theta}{\delta_{5}}\left(\frac{T}{\mathrm{MeV}}\right)^{4}\right| \approx 1
$$

Therefore, for positive helicity neutrinos and negative helicity antineutrinos medium oscillations are the same as vacuum oscillations both in the mixing angle as well as in the oscillation time scales. In this regime of temperature positive helicity neutrinos and antineutrinos are sterile in the sense that these do not interact with the medium.

$$
\text { 2. } \mathbf{m}_{\mathbf{e}}, \mathbf{m}_{\mu} \ll \mathbf{T} \ll \mathbf{M}_{\mathbf{W}}
$$

- case $\mathbf{I}: \omega_{1,2}(k, \lambda)=\lambda\left(k+\frac{M_{1,2}^{2}}{2 k}\right) ; \lambda=+1, h=-1$ and $\lambda=-1, h=+1$ :

This case describes negative helicity neutrinos and positive helicity antineutrinos. Eq. (IV.51) indicates that in this case, $\left|\Delta_{h}\left(\lambda E_{1,2}, k\right) / \delta M^{2}\right| \gg 1$ corresponding to vanishing mixing angle in the medium. Therefore, we obtain

$$
\begin{aligned}
\delta \omega_{1}^{(h)}(k, \lambda)-\delta \omega_{2}^{(h)}(k, \lambda) & \approx \operatorname{sign}\left(\delta M^{2}\right)\left[A_{e}(k)-A_{\mu}(k)-B_{e}(k)+B_{\mu}(k)\right] \\
& =\operatorname{sign}\left(\delta M^{2}\right) \frac{2 G_{F} k T^{2}}{3 \sqrt{2}}\left(\frac{m_{\mu}}{M_{W}}\right)^{2} \\
& \simeq 9.6 \times 10^{-6} \mathrm{eV} \operatorname{sign}\left(\delta M^{2}\right)\left(\frac{k}{\mathrm{MeV}}\right)\left(\frac{T}{\mathrm{GeV}}\right)^{2} .
\end{aligned}
$$

and the ratio of time scales is given by

$$
\frac{\tau_{v}(k)}{\tau_{m}^{(h)}(k, \lambda)} \simeq\left|1+\frac{10^{12}}{\left|\delta_{5}\right|}\left(\frac{k T}{\mathrm{GeV}^{2}}\right)^{2}\right| \gg 1
$$

Hence there is a considerable speed-up in the oscillation time scale in the medium. Again, in this case oscillations are strongly suppressed not only by a vanishingly small mixing angle but also by the rapid dephasing in the medium. 
- case II: $\omega_{1,2}(k, \lambda)=\lambda\left(k+\frac{M_{1,2}^{2}}{2 k}\right) ; \lambda=+1, h=+1$ and $\lambda=-1, h=-1$ :

This case describes positive helicity neutrinos negative helicity antineutrinos. Eq. (IV.52) shows that in this case $\left|\Delta_{h}\left(\lambda E_{1,2}, k\right) / \delta M^{2}\right| \ll 1$, the mixing angle in the medium is the same as in the vacuum. We find for this case

$$
\begin{aligned}
\delta \omega_{1}^{(h)}(k, \lambda)-\delta \omega_{2}^{(h)}(k, \lambda) & \approx-\frac{\bar{M}^{2}}{4 k^{2}} \cos 2 \theta\left[A_{e}(k)-A_{\mu}(k)+B_{e}(k)-B_{\mu}(k)\right] \\
& =\frac{\bar{M}^{2}}{4 k^{2}} \cos 2 \theta \frac{2 G_{F} k T^{2}}{3 \sqrt{2}}\left(\frac{m_{\mu}}{M_{W}}\right)^{2} \\
& \simeq 1.5 \times 10^{-19} \mathrm{eV} \cos 2 \theta\left(\frac{k}{\mathrm{MeV}}\right)^{-1}\left(\frac{T}{\mathrm{GeV}}\right)^{2} .
\end{aligned}
$$

where we have taken $\bar{M} \sim 1 \mathrm{eV}$. The ratio of time scales in this case is given by

$$
\frac{\tau_{v}(k)}{\tau_{m}^{(h)}(k, \lambda)} \sim\left|1+\frac{3 \times 10^{-8} \cos 2 \theta}{\delta_{5}}\left(\frac{T}{\mathrm{GeV}}\right)^{2}\right| \approx 1
$$

Again in this temperature regime we find that positive helicity neutrinos and negative helicity antineutrinos are almost "sterile" in the sense that neither the mixing angle nor the oscillation time scales receive substantial loop corrections. Thus the combined analysis of mixing angle and propagation frequencies in the medium in the temperature regime under consideration indicates that in medium corrections for positive helicity neutrinos and negative helicity antineutrinos are very small. These degrees of freedom are effectively sterile in that their dynamics is (almost) the same as in the vacuum.

\section{LAPLACE TRANSFORM AND REAL-TIME EVOLUTION}

The main purpose to obtain the Dirac equation in real time is to study the oscillations of neutrinos in the medium as an initial value problem. As described in section $\amalg$ this is achieved by adiabatically switching the sources $\eta, \bar{\eta}$ from $t=-\infty$ and switching them off at $t=0$. The adiabatic switching on of the sources induces an expectation value, which evolves in the absence of sources for $t>0$, after the external source has been switched off. It is convenient to write the effective Dirac eq. (II.17) in terms of spatial Fourier transforms. Using the results of the appendix (A3) we find

$$
\left[\left(i \gamma^{0} \frac{\partial}{\partial t}-\vec{\gamma} \cdot \vec{k}\right) \delta_{a b}-M_{a b}+\Sigma_{a b}^{t a d} L\right] \psi_{b}(\vec{k}, t)+\int_{-\infty}^{t} d t^{\prime} \Sigma_{a b}\left(\vec{k}, t-t^{\prime}\right) L \psi_{b}\left(\vec{k}, t^{\prime}\right)=-\eta_{a}(\vec{k}, t)
$$

where the results of appendix (A3) yield

$$
\Sigma\left(\vec{k}, t-t^{\prime}\right)=i \int_{-\infty}^{\infty} \frac{d k_{0}}{\pi} \operatorname{Im} \Sigma\left(\vec{k}, k_{0}\right) e^{-i k_{0}\left(t-t^{\prime}\right)} \quad, \quad \Sigma\left(\vec{k}, k_{0}\right)=\Sigma_{W}\left(\vec{k}, k_{0}\right)+\Sigma_{Z}\left(\vec{k}, k_{0}\right)
$$

For an external Grassmann valued source adiabatically switched on at $t=-\infty$ and off at $t=0$

$$
\eta_{a}(\vec{k}, t)=\eta_{a}(\vec{k}, 0) e^{\epsilon t} \theta(-t) \quad, \quad \epsilon \rightarrow 0^{+} .
$$

It is straightforward to confirm that the solution of the Dirac eq. (V.1) for $t<0$ is given by

$$
\psi_{a}(\vec{k}, t<0)=\psi_{a}(\vec{k}, 0) e^{\epsilon t}
$$

Inserting this ansatze into the equation (V.1) it is straightforward to check that it is indeed a solution with a linear relation between $\psi_{a}(\vec{k}, 0)$ and $\eta_{a}(\vec{k}, 0)$. This relation can be used to obtain $\psi_{a}(\vec{k}, 0)$ from $\eta_{a}(\vec{k}, 0)$, or alternatively, for a given initial value of the field at $t=0$ to find the source $\eta_{a}(\vec{k}, 0)$ that prepares this initial value. For $t>0$ the source term vanishes, the non-local integral in eq. V.1 can be split into an integral from $t=-\infty$ to $t=0$ plus an 
integral from $t=0$ to $t$. In the first integral corresponding to $t<0$ we insert the solution eq.(V.4) and obtain the following equation valid for $t>0$

$$
\begin{aligned}
& {\left[\left(i \gamma^{0} \frac{\partial}{\partial t}-\vec{\gamma} \cdot \vec{k}\right) \delta_{a b}-M_{a b}+\Sigma_{a b}^{t a d} L\right] \psi_{b}(\vec{k}, t)+\int_{0}^{t} d t^{\prime} \Sigma_{a b}\left(\vec{k}, t-t^{\prime}\right) L \psi_{b}\left(\vec{k}, t^{\prime}\right) } \\
= & -\int_{-\infty}^{+\infty} \frac{d k_{0}}{\pi} \frac{\operatorname{Im} \Sigma_{a b}\left(\vec{k}, k_{0}\right)}{k_{0}} e^{-i k_{0} t} L \psi_{b}(\vec{k}, 0) .
\end{aligned}
$$

This equation can be solved by Laplace transform. Introduce the Laplace transforms

$$
\widetilde{\psi}(\vec{k}, s)=\int_{0}^{\infty} d t e^{-s t} \psi(\vec{k}, t) \quad, \quad \widetilde{\Sigma}(\vec{k}, s)=\int_{0}^{\infty} d t e^{-s t} \Sigma(\vec{k}, t)=\int_{-\infty}^{+\infty} \frac{d k_{0}}{\pi} \frac{\operatorname{Im} \Sigma\left(\vec{k}, k_{0}\right)}{k_{0}-i s}
$$

where we have used eq. (V.2) to obtain the Laplace transform of the self-energy, which leads to the analyticity relation (see Eq. (III.1) $)$,

$$
\widetilde{\Sigma}(\vec{k}, s)=\Sigma(\vec{k}, \omega=i s-i \epsilon)
$$

In terms of Laplace transforms the equation of motion becomes the following algebraic equation

$$
\left[\left(i \gamma^{0} s-\vec{\gamma} \cdot \vec{k}\right) \delta_{a b}-M_{a b}+\Sigma_{a b}^{t a d} L+\widetilde{\Sigma}_{a b}(\vec{k}, s) L\right] \widetilde{\psi}_{b}(\vec{k}, s)=i\left\{\gamma^{0} \delta_{a b}+\frac{1}{i s}\left[\widetilde{\Sigma}_{a b}\left(\vec{k}_{,} s\right)-\widetilde{\Sigma}_{a b}(\vec{k}, 0)\right] L\right\} \psi_{b}(\vec{k}, 0)
$$

Consistently with the expansion of the self-energy in frequency and momentum up to order $\omega / M_{W}$, and using eq.(V.7), we replace the expression in the bracket in eq. (D.8) by

$$
\left.\frac{1}{i s}\left[\widetilde{\Sigma}_{a b}(\vec{k}, s)-\widetilde{\Sigma}_{a b}(\vec{k}, 0)\right]\right|_{s=0}=\left.\frac{\partial \Sigma(\vec{k}, \omega)}{\partial \omega}\right|_{\omega=0} \equiv \Sigma^{\prime}(\vec{k}, 0) \text {. }
$$

Using the representation (III.15) for the real part of the self-energy, the eq. (V.8) can be written as

$$
\left[\left(\gamma^{0} i s-\vec{\gamma} \cdot \vec{k}\right) \mathbb{1}-\mathbf{M}+\gamma^{0} \widetilde{\mathbb{A}}(s) L-\vec{\gamma} \cdot \widehat{\mathbf{k}} \mathbb{B}(k) L\right] \widetilde{\psi_{b}}(\vec{k}, s)=i \gamma^{0}\left[\mathbb{1}+\mathbb{A}^{\prime}(0) L\right] \psi_{b}(\vec{k}, 0),
$$

where

$$
\widetilde{\mathbb{A}}(s)=\mathbb{A}(\omega=i s) ; \quad \mathbb{A}^{\prime}(0)=\left.\frac{d \mathbb{A}}{d \omega}\right|_{\omega=0} .
$$

The real time evolution is obtained by the inverse Laplace transform,

$$
\psi(\vec{k}, t)=\int_{\Gamma} \frac{d s}{2 \pi i} \widetilde{\psi}(\vec{k}, s) e^{s t},
$$

where $\Gamma$ is the Bromwich contour in the complex $s$ plane running parallel to the imaginary axis to the right of all the singularities of the function $\widetilde{\psi}(\vec{k}, s)$ and closing on a large semicircle to the left. We now follow the same steps as in section IV namely projecting onto right and left components and onto helicity eigenstates. After straightforward manipulations we arrive at the following set of equations

$$
\left[-\left(s^{2}+k^{2}\right) \mathbb{1}+(i s-\hat{h}(\widehat{\mathbf{k}}) k)(\widetilde{\mathbf{A}}+\hat{h}(\widehat{\mathbf{k}}) \mathbb{B})-\mathbb{M}^{2}\right] \widetilde{\psi}_{L}(\vec{k}, s)=i \gamma^{0} \mathbf{M} \psi_{R}(\vec{k}, 0)+i(i s-\hat{h}(\widehat{\mathbf{k}}) k) \mathbb{D} \psi_{L}(\vec{k}, 0)
$$

where $\mathbb{D}=\mathbb{1}+\mathbb{A}^{\prime}(0)$, and

$$
\widetilde{\psi}_{R}(\vec{k}, s)=-\frac{i s+\hat{h}(\widehat{\mathbf{k}}) k}{s^{2}+k^{2}}\left[\operatorname{IM} \gamma^{0} \widetilde{\psi}_{L}(\vec{k}, s)+i \psi_{R}(\vec{k}, 0)\right] .
$$

We now follow the same steps as above to separate the Dirac and flavor structures by introducing the flavor doublets $\widetilde{\varphi}(\vec{k}, s), \widetilde{\xi}(\vec{k}, s)$ which are the Laplace transform of the flavor doublets $\varphi(\vec{k}, t), \xi(\vec{k}, t)$ introduced in the expansion of the Dirac spinors in eqs. (IV.12)-(IV.13), projecting onto the Weyl spinors eigenstates of helicity, the above equations become

$$
\left[-\left(s^{2}+k^{2}\right) \mathbb{1}+(i s-h k)(\widetilde{\mathbb{A}}+h \mathbb{B})-\mathbf{M}^{2}\right] \widetilde{\varphi}^{(h)}(\vec{k}, s)=-i \operatorname{M} \xi^{(h)}(\vec{k}, 0)+i(i s-h k) \mathbb{D} \varphi^{(h)}(\vec{k}, 0)
$$




$$
\widetilde{\xi}^{(h)}(\vec{k}, s)=-\frac{i s+h k}{s^{2}+k^{2}}\left[-\mathrm{M} \widetilde{\varphi}^{(h)}(\vec{k}, s)+i \xi^{(h)}(\vec{k}, 0)\right]
$$

The solution to eq.

$$
\widetilde{\varphi}^{(h)}(\vec{k}, s)=\widetilde{\mathbb{S}}^{(h)}(k, s)\left[-i \mathrm{M} \xi^{(h)}(\vec{k}, 0)+i(i s-h k) \mathbb{D} \varphi^{(h)}(\vec{k}, 0)\right],
$$

where the propagator is given by

$$
\widetilde{\mathbb{S}}^{h}(k, s)=\frac{1}{\alpha_{h}^{2}(k, s)-\beta_{h}^{2}(k, s)}\left(\begin{array}{cc}
\alpha_{h}(k, s)+\beta(k, s) \cos 2 \theta_{m}^{(h)} & -\beta_{h}(k, s) \sin 2 \theta_{m}^{(h)} \\
-\beta_{h}(k, s) \sin 2 \theta_{m}^{(h)} & \alpha_{h}(k, s)-\beta_{h}(k, s) \cos 2 \theta_{m}^{(h)}
\end{array}\right)
$$

in which it will prove convenient to introduce the following quantities

$$
\begin{gathered}
\alpha_{h}(k, s)=\left[\omega^{2}-k^{2}+\frac{1}{2} S_{h}(\omega, k)-\frac{1}{2}\left(M_{1}^{2}+M_{2}^{2}\right)\right]_{\omega=i s-i \epsilon} \\
\beta_{h}(k, s)=\left.\frac{1}{2} \delta M^{2}\left[\left(\cos 2 \theta-\frac{\Delta_{h}(\omega, k)}{\delta M^{2}}\right)^{2}+\sin ^{2} 2 \theta\right]^{\frac{1}{2}}\right|_{\omega=i s-i \epsilon} .
\end{gathered}
$$

The inverse Laplace transform eq. (V.12) can be done straightforwardly, the singularities of $\tilde{\varphi}^{(h)}(\vec{k}, s)$ in the complex $s$ plane are determined by the singularities of the propagator $\widetilde{\mathbb{S}}^{(h)}(k, s)$. Up to the order in weak interactions considered here, these singularities are isolated poles along the imaginary axis at the positions $s=-i \omega_{a}^{(h)}(k, \pm)$ given by eq. (IV.36)-(IV.39). As a relevant example of the real time evolution, let us consider that the initial state corresponds to a wave-packet of left-handed electron neutrinos of arbitrary helicity $h$, with no muon neutrinos. This could, for example, be the case relevant for nucleosynthesis in which a neutron beta decays at the initial time. In this case

$$
\varphi^{(h)}(\vec{k}, 0)=\nu_{e}^{(h)}(\vec{k})\left(\begin{array}{l}
1 \\
0
\end{array}\right) \quad, \quad \xi^{(h)}(\vec{k}, 0)=\left(\begin{array}{l}
0 \\
0
\end{array}\right)
$$

and we find

$$
\begin{gathered}
\widetilde{\varphi}^{(h)}(\vec{k}, s)=\nu_{e}^{(h)}(\vec{k}) \frac{i(i s-h k)\left[1+A_{e}^{\prime}(0)\right]}{\alpha_{h}^{2}(k, s)-\beta_{h}^{2}(k, s)}\left(\begin{array}{c}
\alpha_{h}(k, s)+\beta_{h}(k, s) \cos \left(2 \theta_{m}^{(h)}\right) \\
-\beta_{h}(k, s) \sin \left(2 \theta_{m}^{(h)}\right)
\end{array}\right), \\
\widetilde{\xi}^{(h)}(\vec{k}, s)=-\nu_{e}^{(h)}(\vec{k}) \frac{i\left[1+A_{e}^{\prime}(0)\right]}{\alpha_{h}^{2}(k, s)-\beta_{h}^{2}(k, s)} \mathrm{M}\left(\begin{array}{c}
\alpha_{h}(k, s)+\beta_{h}(k, s) \cos \left(2 \theta_{m}^{(h)}\right) \\
-\beta_{h}(k, s) \sin \left(2 \theta_{m}^{(h)}\right)
\end{array}\right) .
\end{gathered}
$$

In order to avoid cluttering the notation, we have not included the frequency argument in the mixing angle in the medium $\theta_{m}^{(h)}$ but such dependence should be understood throughout.

The term $\left[\alpha_{h}(k, s)-\beta_{h}(k, s)\right]^{-1}$ features poles at $s=-i \omega_{1}(k, \pm)$ and the term $\left[\alpha_{h}(k, s)+\beta_{h}(k, s)\right]^{-1}$ features poles at $s=-i \omega_{2}(k, \pm)$.

We neglect terms of order $G_{F} T^{4} / M_{W}^{2} \sim\left(T / M_{W}\right)^{4} \ll 1$ since in the regime in which the approximations are valid $T \ll M_{W}$. The residues of these poles are respectively $2 \omega_{1,2}(k, \pm)$ and the inverse Laplace transform yield within these approximations,

$$
\varphi^{(h)}(\vec{k}, t)=\nu_{e}^{(h)}(\vec{k}) \sum_{\lambda= \pm}\left[\frac{\omega_{1}^{(h)}(k, \lambda)-h k}{4 \omega_{1}^{(h)}(k, \lambda)}\left(\begin{array}{c}
1+C_{1, \lambda}^{(h)} \\
-S_{1, \lambda}^{(h)}
\end{array}\right) e^{-i \omega_{1}^{(h)}(k, \lambda) t}+\frac{\omega_{2}^{(h)}(k, \lambda)-h k}{4 \omega_{2}^{(h)}(k, \lambda)}\left(\begin{array}{c}
1-C_{2, \lambda}^{(h)} \\
S_{2, \lambda}^{(h)}
\end{array}\right) e^{-i \omega_{2}^{(h)}(k, \lambda) t}\right]
$$




$$
\xi^{(h)}(\vec{k}, t)=-\nu_{e}^{(h)}(\vec{k}) \sum_{\lambda= \pm}\left[\frac{\mathrm{M}}{4 \omega_{1}^{(h)}(k, \lambda)}\left(\begin{array}{c}
1+C_{1, \lambda}^{(h)} \\
-S_{1, \lambda}^{(h)}
\end{array}\right) e^{-i \omega_{1}^{(h)}(k, \lambda) t}+\frac{\mathrm{IM}}{4 \omega_{2}^{(h)}(k, \lambda)}\left(\begin{array}{c}
1-C_{2, \lambda}^{(h)} \\
S_{2, \lambda}^{(h)}
\end{array}\right) e^{-i \omega_{2}^{(h)}(k, \lambda) t}\right] .
$$

For economy of notation, we introduce the following shorthand

$$
C_{a, \lambda}^{(h)} \equiv \cos \left[2 \theta_{m}^{(h)}\left(\omega_{a}^{(h)}(k, \lambda)\right)\right] \quad, \quad S_{a, \lambda}^{(h)} \equiv \sin \left[2 \theta_{m}^{(h)}\left(\omega_{a}^{(h)}(k, \lambda)\right)\right]
$$

for $a=1,2$, helicity components $h= \pm$ and positive and energy components $\lambda= \pm$.

The above expressions yield a direct comparison with the usual oscillation formulae in the literature. To leading order we set $\omega_{1,2}(k, \lambda) \approx \lambda k\left(1+M_{1,2}^{2} / 2 k^{2}\right)$ in the prefactors in the expressions above thereby neglecting terms of order $G_{F}$. Since the dependence of the mixing angle on the frequency and momentum appears at order $G_{F}$, we can set $\omega_{1,2}(k, \lambda) \approx \lambda k$ in the argument of the mixing angles, therefore to leading order $C_{1, \lambda}^{(h)}=C_{2, \lambda}^{(h)} \equiv C_{\lambda}^{(h)}$ and $S_{1, \lambda}^{(h)}=S_{2, \lambda}^{(h)} \equiv S_{\lambda}^{(h)}$. With these approximations, for an initial left handed electron state of helicity $h=\mp$ we find

$$
\begin{aligned}
\varphi^{-}(\vec{k}, t) & =\nu_{e}^{-}(\vec{k})\left[\frac{1}{2}\left(\begin{array}{c}
1+C_{+}^{-} \\
-S_{+}^{-}
\end{array}\right) e^{-i \omega_{1}^{-}(k,+) t}+\frac{1}{2}\left(\begin{array}{c}
1-C_{+}^{-} \\
S_{+}^{-}
\end{array}\right) e^{-i \omega_{2}^{-}(k,+) t}\right. \\
& \left.+\frac{M_{1}^{2}}{8 k^{2}}\left(\begin{array}{c}
1+C_{-}^{-} \\
-S_{-}^{-}
\end{array}\right) e^{-i \omega_{1}^{-}(k,-) t}+\frac{M_{2}^{2}}{8 k^{2}}\left(\begin{array}{c}
1-C_{-}^{-} \\
S_{-}^{-}
\end{array}\right) e^{-i \omega_{2}^{-}(k,-) t}\right] \\
\varphi^{+}(\vec{k}, t) & =\nu_{e}^{+}(\vec{k})\left[\frac{M_{1}^{2}}{8 k^{2}}\left(\begin{array}{c}
1+C_{+}^{+} \\
-S_{+}^{+}
\end{array}\right) e^{-i \omega_{1}^{+}(k,+) t}+\frac{M_{2}^{2}}{8 k^{2}}\left(\begin{array}{c}
1-C_{+}^{+} \\
S_{+}^{+}
\end{array}\right) e^{-i \omega_{2}^{+}(k,+) t}\right. \\
& \left.+\frac{1}{2}\left(\begin{array}{c}
1+C_{-}^{+} \\
-S_{-}^{+}
\end{array}\right) e^{-i \omega_{1}^{+}(k,-) t}+\frac{1}{2}\left(\begin{array}{c}
1-C_{-}^{+} \\
S_{-}^{+}
\end{array}\right) e^{-i \omega_{2}^{+}(k,-) t}\right]
\end{aligned}
$$

The exponentials $e^{\mp i \omega(k) t}$ correspond to positive energy $(-)$ neutrino and positive energy $(+)$ antineutrino components respectively. Therefore, the expressions above reveal that for negative helicity the relevant components in the relativistic limit correspond to positive energy neutrinos, while for positive helicity they correspond to positive energy antineutrinos.

The upper component of the expressions above correspond to wavepackets of negative and positive helicity respectively for a left handed electron neutrino, namely $\nu_{e}^{\mp}(\vec{k}, t)$ while the lower components correspond to a left handed muon neutrino, namely $\nu_{\mu}^{\mp}(\vec{k}, t)$.

For the right handed components $\xi$ the leading order can be obtained by setting $\mathbb{M} \approx \bar{M} \mathbb{1}$ thereby neglecting terms of order $\delta M^{2} / \bar{M}^{2}$. This approximation yields,

$$
\begin{aligned}
\xi^{\mp}(\vec{k}, t)= & -\nu_{e}^{\mp}(\vec{k}) \frac{\bar{M}}{4 k}\left[\left(\begin{array}{c}
1+C_{+}^{\mp} \\
-S_{+}^{\mp}
\end{array}\right) e^{-i \omega_{1}^{\mp}(k,+) t}+\left(\begin{array}{c}
1-C_{+}^{\mp} \\
S_{+}^{\mp}
\end{array}\right) e^{-i \omega_{2}^{\mp}(k,+) t}\right. \\
& \left.-\left(\begin{array}{c}
1+C_{-}^{\mp} \\
-S_{-}^{\mp}
\end{array}\right) e^{-i \omega_{1}^{\mp}(k,-) t}-\left(\begin{array}{c}
1-C_{-}^{\mp} \\
S_{-}^{\mp}
\end{array}\right) e^{-i \omega_{2}^{\mp}(k,-) t}\right] .
\end{aligned}
$$

The upper and lower components correspond to wavepackets for right handed negative and positive helicity electron and muon neutrinos respectively.

From the expression (V.27) we can read off the probability for relativistic left handed, negative helicity electron and muon neutrinos as a function of time,

$$
\begin{aligned}
& \left|\nu_{e, L}^{-}(\vec{k}, t)\right|^{2}=\left|\nu_{e}^{-}(\vec{k})\right|^{2}\left\{1-\sin ^{2}\left[2 \theta_{m}^{-}(k)\right] \sin ^{2}\left(\frac{1}{2} \Delta \omega^{-}(k,+) t\right)+\mathcal{O}\left(\frac{M_{1,2}^{2}}{k^{2}}\right)\right\} \\
& \left|\nu_{\mu, L}^{-}(\vec{k}, t)\right|^{2}=\left|\nu_{e}^{-}(\vec{k})\right|^{2}\left\{\sin ^{2}\left[2 \theta_{m}^{-}(k)\right] \sin ^{2}\left(\frac{1}{2} \Delta \omega^{-}(k,+) t\right)+\mathcal{O}\left(\frac{M_{1,2}^{2}}{k^{2}}\right)\right\} .
\end{aligned}
$$


The probability for left handed relativistic positive helicity electron and muon antineutrinos as a function of time are read off from eq. (D.28)

$$
\begin{aligned}
\left|\nu_{e, L}^{+}(\vec{k}, t)\right|^{2} & =\left|\nu_{e}^{+}(\vec{k})\right|^{2}\left\{1-\sin ^{2}\left[2 \theta_{m}^{+}(-k)\right] \sin ^{2}\left(\frac{1}{2} \Delta \omega^{+}(k,-) t\right)+\mathcal{O}\left(\frac{M_{1,2}^{2}}{k^{2}}\right)\right\}, \\
\left|\nu_{\mu, L}^{+}(\vec{k}, t)\right|^{2} & =\left|\nu_{e}^{+}(\vec{k})\right|^{2}\left\{\sin ^{2}\left[2 \theta_{m}^{+}(-k)\right] \sin ^{2}\left(\frac{1}{2} \Delta \omega^{+}(k,-) t\right)+\mathcal{O}\left(\frac{M_{1,2}^{2}}{k^{2}}\right)\right\},
\end{aligned}
$$

where

$$
\Delta \omega^{ \pm}(k, \pm)=\frac{\delta M^{2}}{2 k}+\delta \omega_{1}^{ \pm}(k, \pm)-\delta \omega_{2}^{ \pm}(k, \pm) .
$$

The corrections $\delta \omega_{a}^{ \pm}(k, \pm)$ had been studied in detail in section IVC above. Finally, eq. (V.29) determines the probabilities of finding right handed positive and negative helicities neutrinos as a function of time. This equation makes manifest that this probability is suppressed by a factor $\bar{M} / k$ with respect to the left handed component, indeed it is the mass term that is responsible for generating a right handed component from a left handed one and must therefore be suppressed by one power of the ratio $M / k$. For a typical neutrino momentum $k \gtrsim 1 \mathrm{MeV}$ this suppression factor is of order $10^{-6}$. Eqs. (V.24)- $\mathbf{V . 2 5}$ provide a complete field theoretical description of oscillations in real time. Eqs.(V.30-(V.31) are obviously reminiscent of the familiar oscillation equations obtained in the simplified quantum mechanical two level system, however there are some important aspects that must be highlighted, namely, the field theoretical formulation introduced here led directly to these oscillation formulae in terms of the mixing angles in the medium and the correct oscillation frequencies that include the quantum loop corrections. Furthermore, the oscillation formulae obtained above reveal the nature of the approximations and allow a consistent inclusion of higher order effects as well as describe the oscillation of all helicity components as well as the dynamics of the right handed component. The usual oscillation formula obtained within the single particle quantum mechanical description emerge cleanly in suitable limits and the nature of the corrections is readily manifest.

\section{CONCLUSIONS, AND FURTHER QUESTIONS:}

We have provided a systematic treatment of neutrino oscillations and mixing directly from quantum field theory in real time in a regime of temperature and density relevant for early Universe cosmology prior to big bang nucleosynthesis. While we have focused on two flavors (electron and muon) of Dirac neutrinos the formulation can be generalized straightforwardly to more flavors and to Majorana-Dirac mass matrices.

We have obtained the medium corrections to the dispersion relations and mixing angles of propagating neutrinos. Implementing methods from real time non-equilibrium quantum field theory at finite temperature and density we have systematically obtained the equations of motion for the neutrino field and studied the real time evolution as an initial value problem. The major advantage of this approach, as compared to the usual approach based on single particle quantum mechanics is that it consistently and systematically includes the medium corrections to the dispersion relations and mixing angles directly into the real time evolution and treats left and right handed fields and both helicity components on equal footing. We have argued that collisional relaxation yields thermalization of neutrinos in flavor eigenstates for temperature $T \gtrsim 5-10 \mathrm{Mev}$ for which the relaxation time scale via weak interactions is shorter than the oscillation time scale. Assuming the validity of this argument, we obtained the neutrino self-energies up to one-loop including the asymmetries from leptons, neutrinos, hadrons and quarks, as well as non-local (in space-time) terms arising from the expansion of the self-energy loop in the external frequency and momentum. We have consider these non-local terms up to leading order in $\omega / M_{W} ; k / M_{W}$ since these terms are of the same order of or larger than the contribution from the asymmetries for $T \gtrsim 5 \mathrm{MeV}$ if all asymmetries are of the same order as the baryon asymmetry. This is yet another indication that this is an important temperature regime in the early Universe.

Our main results are summarized as follows:

- Implementing the methods from non-equilibrium real-time field theory at finite temperature and density we obtained the equations of motion for the neutrino fields in linear response. This formulation includes consistently the self-energy loop corrections to the dispersion relations and mixing angles in the medium and treat left and right handed fields with both helicity components on equal footing.

- We have focused on a temperature regime prior to nucleosynthesis $T \gtrsim 5-10 \mathrm{MeV}$ in which we argued that neutrinos are thermalized as flavor eigenstates. We studied two different temperature regimes: $m_{e} \ll T \ll m_{\mu}$ within which we have shown that there is the possibility of resonant oscillations of test neutrinos, and $m_{e}, m_{\mu} \ll$ $T \ll M_{W}$ within which the mixing angle for active neutrinos effectively vanishes. 
- An expansion of the self-energy in terms of the neutrino frequency and momentum is carried out to lowest order in $\omega / M_{W} ; k / M_{W}$ thus extracting the leading non-local (in space-time) contributions. We find a new contribution which cannot be identified with an effective potential. The mixing angles and propagation frequencies in the medium are found to be helicity dependent. It has been recently pointed out [46] that a neutrino helicity asymmetry could be a very important ingredient in successful see-saw models of thermal leptogenesis.

- If the lepton and quark asymmetries are of the same order as the baryon asymmetry in the early Universe, we have shown that the non-local (in space-time) terms in the self-energies dominate over the asymmetry for typical energies of neutrinos in the plasma for $T \gtrsim 3-5 \mathrm{MeV}$.

- The oscillation time scale in the medium is slowed-down near the resonance, becoming substantially longer than in the vacuum for small vacuum mixing angle. For high energy neutrinos off-resonance the mixing angle becomes vanishingly small and the oscillation time scale speeds-up as compared to the vacuum. At high temperature, in the region $T \gg m_{e}, m_{\mu}$ the mixing angle for active neutrinos effectively vanishes and there is a considerable speed-up of oscillations, which are then suppressed by a vanishingly small mixing angle and a rapid dephasing.

- We have obtained the general equations of motion for initially prepared wave packets of neutrinos of arbitrary chirality and helicity. These equations reduce to the familiar oscillation formulae for ultrarelativistic negative helicity neutrinos, but with the bonus that they consistently include the mixing angles and the oscillation frequencies in the medium. These equations not only yield the familiar ones but also quantify the magnitude of the corrections. Furthermore these equations also describe the evolution of right handed neutrinos (of either helicity) which is a consequence of a non-trivial mass matrix and usually ignored in the usual formulation.

Further questions: The next step in the program is to provide a solid assessment of the reliability of the argument that suggests that for $T \gtrsim 10 \mathrm{MeV}$ neutrinos thermalize as flavor eigenstates. The phenomenon of slowdown of oscillations near a resonance found in this work, suggests that this argument is consistent at least near resonances. We are currently studying the relaxational dynamics by including two loop corrections to the self-energy which account for collisional processes in the medium. We are also studying how to obtain the kinetic equations that describe simultaneously oscillations and relaxation systematically within a field theory approach that accounts for the subtle aspects of the Fock representation necessary to understand the concept of the flavor distribution functions [37].

\section{Acknowledgments}

D.B. thanks the US NSF for the support under grant PHY-0242134 and A. Dolgov for discussions. C.M.Ho. acknowledges the support through the Andrew Mellon Foundation.

\section{APPENDIX A: REAL-TIME PROPAGATORS AND SELF-ENERGIES.}

\section{Fermions}

Consider a generic fermion field $f(\vec{x}, t)$ of mass $m_{f}$. The Wightmann and Green's functions at finite temperature are given as

$$
\begin{aligned}
& i S_{\alpha, \beta}^{>}\left(\vec{x}-\vec{x}^{\prime}, t-t^{\prime}\right)=\left\langle f_{\alpha}(\vec{x}, t) \bar{f}_{\beta}\left(\vec{x}^{\prime}, t^{\prime}\right)\right\rangle=\frac{1}{V} \sum_{\vec{p}} e^{i \vec{p} \cdot\left(\vec{x}-\vec{x}^{\prime}\right)} i S_{\alpha, \beta}^{>}\left(\vec{p}, t-t^{\prime}\right), \\
& i S_{\alpha, \beta}^{<}\left(\vec{x}-\vec{x}^{\prime}, t-t^{\prime}\right)=-\left\langle\bar{f}_{\beta}\left(\vec{x}^{\prime}, t^{\prime}\right) f_{\alpha}(\vec{x}, t)\right\rangle=\frac{1}{V} \sum_{\vec{p}} e^{i \vec{p} \cdot\left(\vec{x}-\vec{x}^{\prime}\right)} i S_{\alpha, \beta}^{<}\left(\vec{p}, t-t^{\prime}\right),
\end{aligned}
$$

where $\alpha, \beta$ are Dirac indices and $V$ is the quantization volume.

The real-time Green's functions along the forward $(+)$ and backward $(-)$ time branches are given in terms of these Wightmann functions as

$$
\begin{aligned}
& \left\langle f_{\alpha}^{(+)}(\vec{x}, t) \bar{f}_{\beta}^{(+)}\left(\vec{x}^{\prime}, t^{\prime}\right)\right\rangle=i S^{++}\left(\vec{x}-\vec{x}^{\prime}, t-t^{\prime}\right)=i S^{>}\left(\vec{x}-\vec{x}^{\prime}, t-t^{\prime}\right) \Theta\left(t-t^{\prime}\right)+i S^{<}\left(\vec{x}-\vec{x}^{\prime}, t-t^{\prime}\right) \Theta\left(t^{\prime}-t\right)(\mathrm{A} \\
& \left\langle f_{\alpha}^{(+)}(\vec{x}, t) \bar{f}_{\beta}^{(-)}\left(\vec{x}^{\prime}, t^{\prime}\right)\right\rangle=i S^{+-}\left(\vec{x}-\vec{x}^{\prime}, t-t^{\prime}\right)=i S^{<}\left(\vec{x}-\vec{x}^{\prime}, t-t^{\prime}\right)
\end{aligned}
$$


At finite temperature $T$, it is straightforward to obtain these correlation functions by expanding the free fermion fields in terms of Fock creation and annihilation operators and massive spinors. In a $C P$ asymmetric medium, the chemical potential $\mu_{f}$ for the fermion $f$ is non-zero. Particles and anti-particles obey the following Fermi-Dirac distribution functions respectively

$$
N_{f}\left(p_{0}\right)=\frac{1}{e^{\left(p_{0}-\mu_{f}\right) / T}+1} \quad, \quad \bar{N}_{f}\left(p_{0}\right)=\frac{1}{e^{\left(p_{0}+\mu_{f}\right) / T}+1} .
$$

The fermionic propagators are conveniently written in a dispersive form

$$
\begin{aligned}
& i S_{\alpha, \beta}^{>}\left(\vec{p}, t-t^{\prime}\right)=\int_{-\infty}^{\infty} d p_{0} \rho_{\alpha, \beta}^{>}\left(\vec{p}, p_{0}\right) e^{-i p_{0}\left(t-t^{\prime}\right)}, \\
& i S_{\alpha, \beta}^{<}\left(\vec{p}, t-t^{\prime}\right)=\int_{-\infty}^{\infty} d p_{0} \rho_{\alpha, \beta}^{<}\left(\vec{p}, p_{0}\right) e^{-i p_{0}\left(t-t^{\prime}\right)},
\end{aligned}
$$

where we have

$$
\begin{aligned}
& \rho_{\alpha, \beta}^{>}\left(\vec{p}, p_{0}\right)=\frac{\gamma^{0} p_{0}-\vec{\gamma} \cdot \vec{p}+m_{f}}{2 p_{0}}\left[1-N_{f}\left(p_{0}\right)\right] \delta\left(p_{0}-\omega_{p}\right)+\frac{\gamma^{0} p_{0}-\vec{\gamma} \cdot \vec{p}+m_{f}}{2 p_{0}} \bar{N}_{f}\left(-p_{0}\right) \delta\left(p_{0}+\omega_{p}\right) \\
& \rho_{\alpha, \beta}^{<}\left(\vec{p}, p_{0}\right)=\frac{\gamma^{0} p_{0}-\vec{\gamma} \cdot \vec{p}+m_{f}}{2 p_{0}} N_{f}\left(p_{0}\right) \delta\left(p_{0}-\omega_{p}\right)+\frac{\gamma^{0} p_{0}-\vec{\gamma} \cdot \vec{p}+m_{f}}{2 p_{0}}\left[1-\bar{N}_{f}\left(-p_{0}\right)\right] \delta\left(p_{0}+\omega_{p}\right)
\end{aligned}
$$

with $\omega_{p}=\sqrt{|\vec{p}|^{2}+m_{f}^{2}}$. Using the relation $\bar{N}_{f}\left(-p_{0}\right)=1-N_{f}\left(p_{0}\right)$, we can write

$$
\begin{aligned}
& \rho_{\alpha, \beta}^{>}\left(\vec{p}, p_{0}\right)=\left[1-N_{f}\left(p_{0}\right)\right] \rho_{\alpha, \beta}^{f}\left(\vec{p}, p_{0}\right), \\
& \rho_{\alpha, \beta}^{<}\left(\vec{p}, p_{0}\right)=N_{f}\left(p_{0}\right) \rho_{\alpha, \beta}^{f}\left(\vec{p}, p_{0}\right)
\end{aligned}
$$

where the free fermionic spectral density $\rho^{f}\left(\vec{p}, p_{0}\right)$ is given by

$$
\begin{aligned}
\rho^{f}\left(\vec{p}, p_{0}\right) & =\frac{\not p_{+}}{2 \omega_{p}} \delta\left(p_{0}-\omega_{p}\right)+\frac{\not p_{-}}{2 \omega_{p}} \delta\left(p_{0}+\omega_{p}\right), \\
\not p_{ \pm} & =\gamma^{0} \omega_{p} \mp \vec{\gamma} \cdot \vec{p} \pm m_{f} .
\end{aligned}
$$

\section{Vector Bosons}

Consider a generic real vector boson field $A_{\mu}(\vec{x}, t)$ of mass $M$. In unitary gauge, it can be expanded in terms of Fock creation and annihilation operators of physical states with three polarizations as

$$
A^{\mu}(\vec{x}, t)=\frac{1}{\sqrt{V}} \sum_{\lambda} \sum_{\vec{k}} \frac{\epsilon_{\lambda}^{\mu}(\vec{k})}{\sqrt{2 W_{k}}}\left[a_{\vec{k}, \lambda} e^{-i W_{k} t} e^{i \vec{k} \cdot \vec{x}}+a_{\vec{k}, \lambda}^{\dagger} e^{i W_{k} t} e^{-i \vec{k} \cdot \vec{x}}\right] \quad ; \quad k^{\mu} \epsilon_{\mu, \lambda}(\vec{k})=0
$$

where $W_{k}=\sqrt{|\vec{k}|^{2}+M^{2}}$ and $k^{\mu}$ is on-shell $k^{\mu}=\left(W_{k}, \vec{k}\right)$. The three polarization vectors are such that

$$
\sum_{\lambda=1}^{3} \epsilon_{\lambda}^{\mu}(\vec{k}) \epsilon_{\lambda}^{\nu}(\vec{k})=P^{\mu \nu}(\vec{k})=-\left(g^{\mu \nu}-\frac{k^{\mu} k^{\nu}}{M^{2}}\right)
$$

It is now straightforward to compute the Wightmann functions of the vector bosons in a state in which the physical degrees of freedom are in thermal equilibrium at temperature $T$. These are given by

$$
\begin{aligned}
\left\langle A_{\mu}(\vec{x}, t) A_{\nu}\left(\vec{x}^{\prime}, t^{\prime}\right)\right\rangle & =i G_{\mu, \nu}^{>}\left(\vec{x}-\vec{x}^{\prime}, t-t^{\prime}\right), \\
\left\langle A_{\nu}\left(\vec{x}^{\prime}, t^{\prime}\right) A_{\mu}(\vec{x}, t)\right\rangle & =i G_{\mu, \nu}^{<}\left(\vec{x}-\vec{x}^{\prime}, t-t^{\prime}\right),
\end{aligned}
$$

where $G^{<,>}$can be conveniently written as spectral integrals in the form

$$
\begin{aligned}
i G_{\mu, \nu}^{>}\left(\vec{x}-\vec{x}^{\prime}, t-t^{\prime}\right) & =\frac{1}{V} \sum_{\vec{k}} e^{i \vec{k} \cdot\left(\vec{x}-\vec{x}^{\prime}\right)} \int_{-\infty}^{\infty} d k_{0} e^{-i k_{0}\left(t-t^{\prime}\right)}\left[1+N_{b}\left(k_{0}\right)\right] \rho_{\mu \nu}\left(k_{0}, \vec{k}\right) \\
i G_{\mu, \nu}^{<}\left(\vec{x}-\vec{x}^{\prime}, t-t^{\prime}\right) & =\frac{1}{V} \sum_{\vec{k}} e^{i \vec{k} \cdot\left(\vec{x}-\vec{x}^{\prime}\right)} \int_{-\infty}^{\infty} d k_{0} e^{-i k_{0}\left(t-t^{\prime}\right)} N_{b}\left(k_{0}\right) \rho_{\mu \nu}\left(k_{0}, \vec{k}\right)
\end{aligned}
$$


where

$$
N_{b}\left(k_{0}\right)=\frac{1}{e^{k_{0} / T}-1}
$$

and the spectral density is given by

$$
\rho_{\mu \nu}\left(k_{0}, \vec{k}\right)=\frac{1}{2 W_{k}}\left[P_{\mu \nu}(\vec{k}) \delta\left(k_{0}-W_{k}\right)-P_{\mu \nu}(-\vec{k}) \delta\left(k_{0}+W_{k}\right)\right] .
$$

In terms of these Wightmann functions, the real-time correlation functions along the forward and backward time branches are given by

$$
\begin{aligned}
& \left\langle A_{\mu}^{(+)}(\vec{x}, t) A_{\nu}^{(+)}\left(\vec{x}^{\prime}, t^{\prime}\right)\right\rangle=i G_{\mu, \nu}^{>}\left(\vec{x}-\vec{x}^{\prime}, t-t^{\prime}\right) \Theta\left(t-t^{\prime}\right)+i G_{\mu, \nu}^{<}\left(\vec{x}-\vec{x}^{\prime}, t-t^{\prime}\right) \Theta\left(t^{\prime}-t\right), \\
& \left\langle A_{\mu}^{(+)}(\vec{x}, t) A_{\nu}^{(-)}\left(\vec{x}^{\prime}, t^{\prime}\right)\right\rangle=i G_{\mu, \nu}^{<}\left(\vec{x}-\vec{x}^{\prime}, t-t^{\prime}\right) .
\end{aligned}
$$

For the charged vector bosons, the correlation functions can be found simply from those of the real vector boson fields described above by writing the charged fields as linear combinations of two real fields $A^{1,2}$, namely

$$
W_{\mu}^{ \pm}(\vec{x}, t)=\frac{1}{\sqrt{2}}\left[A_{\mu}^{1}(\vec{x}, t) \pm i A_{\mu}^{2}(\vec{x}, t)\right]
$$

It is straightforward to find the correlation function

$$
\left\langle W_{\mu}^{+}(\vec{x}, t) W_{\mu}^{-}\left(\vec{x}^{\prime}, t^{\prime}\right)\right\rangle=G_{\mu \nu}^{>}\left(\vec{x}-\vec{x}^{\prime}, t-t^{\prime}\right),
$$

and similarly for the other necessary Wightmann and Green's functions.

\section{Retarded Self-Energies for Charged and Neutral Current Interactions}

The diagrams for the one-loop retarded self-energy from charged current interactions are displayed in Fig. (2). A straightforward calculation yields for the charged current contribution the following result

$\Sigma_{r e t}^{C C}\left(\vec{x}-\vec{x}^{\prime}, t-t^{\prime}\right)=\frac{i g^{2}}{2} R \gamma^{\mu}\left[i S^{++}\left(\vec{x}-\vec{x}^{\prime}, t-t^{\prime}\right) i G_{\mu \nu}^{++}\left(\vec{x}-\vec{x}^{\prime}, t-t^{\prime}\right)-i S^{<}\left(\vec{x}-\vec{x}^{\prime}, t-t^{\prime}\right) i G_{\mu \nu}^{<}\left(\vec{x}-\vec{x}^{\prime}, t-t^{\prime}\right)\right] \gamma^{\nu} L$,

with

$$
R=\frac{1+\gamma^{5}}{2} \quad, \quad L=\frac{1-\gamma^{5}}{2}
$$

A similar result is obtained for the neutral current contribution to the self-energy by simply replacing $g / \sqrt{2} \rightarrow$ $g / 2 \cos \theta_{w}$ and $M_{W} \rightarrow M_{Z}=M_{W} / \cos \theta_{w}$.

Using the representation of the fermion and vector boson propagators given above the retarded self-energy (A.26) can be written as

$$
\Sigma_{r e t}\left(\vec{x}-\vec{x}^{\prime}, t-t^{\prime}\right)=\frac{i}{V} \sum_{\vec{k}} \int_{-\infty}^{\infty} d k_{0} R\left[\bar{\Sigma}_{W}\left(\vec{k}, k_{0}\right)+\bar{\Sigma}_{Z}\left(\vec{k}, k_{0}\right)\right] L e^{i \vec{k} \cdot\left(\vec{x}-\vec{x}^{\prime}\right)} e^{-i k_{0}\left(t-t^{\prime}\right)} \Theta\left(t-t^{\prime}\right),
$$

The contributions from charged and neutral vector bosons are given by

$$
\begin{aligned}
& \bar{\Sigma}_{W}\left(\vec{k}, k_{0}\right)=\frac{g^{2}}{2} \int \frac{d^{3} q}{(2 \pi)^{3}} \int d p_{0} \int d q_{0} \delta\left(p_{0}+q_{0}-k_{0}\right) \gamma^{\mu} \rho^{f}\left(\vec{k}-\vec{q}, p_{0}\right) \rho_{\mu \nu}^{W}(\vec{k})\left(\vec{q}, q_{0}\right) \gamma^{\nu}\left[1-N_{f}\left(p_{0}\right)+N_{b}\left(q_{0}\right)\right] \\
& \bar{\Sigma}_{Z}\left(\vec{k}, k_{0}\right)=\frac{g^{2}}{4 \cos ^{2} \theta_{w}} \int \frac{d^{3} q}{(2 \pi)^{3}} \int d p_{0} \int d q_{0} \delta\left(p_{0}+q_{0}-k_{0}\right) \gamma^{\mu} \rho^{f}\left(\vec{k}-\vec{q}, p_{0}\right) \rho_{\mu \nu}^{Z}\left(\vec{q}, q_{0}\right) \gamma^{\nu}\left[1-N_{f}\left(p_{0}\right)+N_{b}\left(q_{0}\right)\right]
\end{aligned}
$$

where $\rho_{W, Z}\left(\vec{q}, q_{0}\right)$ are the vector boson spectral densities given by eq.A.21 with $M \equiv M_{W, Z}$ respectively. It is clear that $\bar{\Sigma}_{W, Z}\left(\vec{k}, k_{0}\right)$ corresponds to a vector-like theory. 


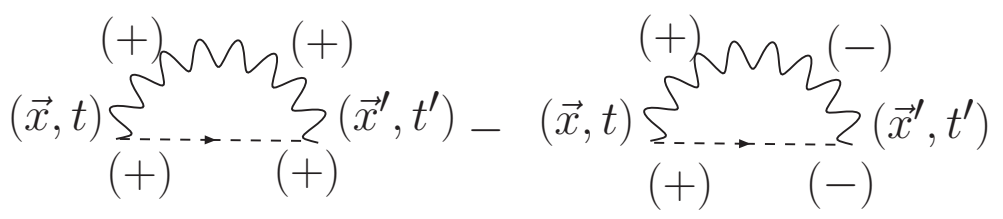

FIG. 2: Retarded self-energy for charged current interactions. The wiggly line is a charged vector boson and the dashed line a lepton. The labels $( \pm)$ correspond to the forward $(+)$ and backward $(-)$ time branches. The corresponding propagators are $i S^{ \pm, \pm}\left(\vec{x}-\vec{x}^{\prime}, t-t^{\prime}\right)$ and $i G_{\mu \nu}^{ \pm \pm}\left(\vec{x}-\vec{x}^{\prime}, t-t^{\prime}\right)$ for leptons and charged bosons respectively.

Using the integral representation of the function $\Theta\left(t-t^{\prime}\right)$, the retarded self-energy can be written in the following simple dispersive form

$$
\begin{gathered}
\Sigma_{r e t}\left(\vec{x}-\vec{x}^{\prime}, t-t^{\prime}\right)=\frac{1}{V} \sum_{\vec{k}} \int_{-\infty}^{\infty} \frac{d \omega}{2 \pi} e^{i \vec{k} \cdot\left(\vec{x}-\vec{x}^{\prime}\right)} e^{-i \omega\left(t-t^{\prime}\right)} R\left[\Sigma_{W}(\vec{k}, \omega)+\Sigma_{Z}(\vec{k}, \omega)\right] L, \\
\Sigma_{W, Z}(\vec{k}, \omega)=\int d k_{0} \frac{\bar{\Sigma}_{W, Z}\left(\vec{k}, k_{0}\right)}{k_{0}-\omega-i \epsilon},
\end{gathered}
$$

where $\epsilon \rightarrow 0^{+}$. Hence, from the above expression, we identify

$$
\bar{\Sigma}_{W, Z}(\vec{k}, \omega)=\frac{1}{\pi} \operatorname{Im} \Sigma_{W, Z}(\vec{k}, \omega) .
$$

Furthermore, since $R\left( \pm m_{f}\right) L=0$, the factor $\pm m_{f}$ in the free fermionic spectral density defined in Eqns. A.12) and (A.13) can be ignored when we compute $R\left[\bar{\Sigma}_{W}(\vec{k}, \omega)+\bar{\Sigma}_{Z}(\vec{k}, \omega)\right] L$. The signature of the the fermion mass $m_{f}$ is only reflected in the factors of $\omega_{p}$ in the spectral density. Ignoring the factor $m_{f}$ from now on, the fermionic spectral density is proportional to the $\gamma$ matrices only and does not feature the identity matrix or $\gamma^{5}$. Therefore, there is the following simplification

$$
R\left[\bar{\Sigma}_{W}(\vec{k}, \omega)+\bar{\Sigma}_{Z}(\vec{k}, \omega)\right] L=\left[\bar{\Sigma}_{W}(\vec{k}, \omega)+\bar{\Sigma}_{Z}(\vec{k}, \omega)\right] L
$$

[1] C. W. Kim and A. Pevsner, Neutrinos in Physics and Astrophysics, Harwood Academic Publishers, USA, 1993.

[2] R. N. Mohapatra and P. B. Pal, Massive Neutrinos in Physics and Astrophysics, World Scientific, Singapore, 2004.

[3] M. Fukugita and T. Yanagida, Physics of Neutrinos and Applications to Astrophysics, Springer-Verlag Berlin Heidelberg 2003.

[4] G. G. Raffelt, Stars as Laboratories for Fundamental Physics, The University of Chicago Press, Chicago, 1996; astro-ph/0302589 New Astron.Rev. 46, 699 (2002); hep-ph/0208024

[5] J. N. Bahcall,Neutrino Astrophysics, Cambridge University Press, Cambridge, 1989.

[6] T. K. Kuo and J. Pantaleone, Rev. of Mod. Phys. 61, 937 (1989).

[7] S.M. Bilenky, C. Giunti, J.A. Grifols, E. Masso, Phys. Rept. 379, 69 (2003); C. Giunti, Found. Phys. Lett. 17, 103 (2004); S. M. Bilenky and C. Giunti, Int. J. Mod. Phys. A16, 3931 (2001); C. Giunti and M. Lavede, hep-ph/0310238 (published in 'Developments in Quantum Physics - 2004', edited by F. Columbus and V. Krasnoholovets, Nova Science Publishers, Inc, NY.) .

[8] A. Yu. Smirnov, Int.J.Mod.Phys. A191180 (2004); hep-ph/0306075 hep-ph/0305106

[9] S. M. Bilenky, hep-ph/0402153 W.M. Alberico, S.M. Bilenky, Phys.Part.Nucl. 35, 297 (2004) [Fiz.Elem.Chast.Atom.Yadra 35, 545 (2004)]; S. M. Bilenky, hep-ph/0210128

[10] W. C. Haxton, in Boulder 1998, Neutrinos in physics and astrophysics, 432, Ed. Paul Langacker, Singapore, World Scientific, 2000, nucl-th/9901076 Wick C. Haxton, Barry R. Holstein, Am. J. Phys. 68, 15 (2000).

[11] W. Grimus, hep-ph/0307149

[12] A. de Gouvea, hep-ph/0411274 TASI lectures on neutrino physics.

[13] L. Wolfenstein, Phys. Rev. D17, 2369 (1978); Phys. Rev. D20, 2634 (1979); Phys. Lett. B194 197 (1987).

[14] S. P. Mikheyev and A. Yu Smirnov, , Yad. Fiz.42, 1441 (1985) (Sov. J. Nucl. Phys. 42, 913 (1985)); Nuovo Cimento C9, 17 (1986); Zh. Eksp. Toer. Fiz. 91, 7 (1986) [Sov. Phys. JETP 64, 4 (1986)]. 
[15] H. A. Bethe, Phys. Rev. Lett. 56, 1305 (1986).

[16] M. J. Savage, R. A. Malaney and G. M. Fuller, Astroph. Jour. 368, 1, 1991; C. Y. Cardall and G. M. Fuller, astro-ph/9702001

[17] A. D. Dolgov, Surveys High Energ.Phys. 17, 91, (2002); Phys. Rept. 370, 333 (2002); Nuovo Cim. 117 B, 1081, (2003); hep-ph/0109155

[18] D. Kirilova and M. Chizhov, astro-ph/0108341 Nucl. Phys. Proc. Suppl. 100, 360 (2001); Phys. Lett. B393, 375 (1997); M. Yu. Khlopov and S. T. Petcov, Phys. Lett B99, 117 (1981).

[19] A.D. Dolgov, F.L. Villante, Nucl.Phys. B679 261, (2004); hep-ph/0310138

[20] G. M. Fuller, W. C. Haxton, G. C. McLaughlin Phys.Rev.D59, 085005 (1999); J. Pantaleone, Phys.Lett. B342, 250 (1995); B. Jegerlehner, F. Neubig, G. Raffelt, Phys.Rev.D54, 1194 (1996).

[21] M. Prakash, J. M. Lattimer, R. F. Sawyer, R. R. Volkas, Ann. Rev. Nucl. Part. Sci. 51, 295 (2001); S. Reddy, M. Prakash, J. M. Lattimer, Phys. Rev. D58, 013009 (1998), M. Prakash, S. Ratkovic and S. I. Dutta, astro-ph/0403038

[22] M. Prakash, J.M. Lattimer, J.A. Pons, A.W. Steiner, S. Reddy, Lect. Notes Phys. 578, 364 (2001), S. Reddy, M. Prakash, nucl-th/9508009

[23] D. G. Yakovlev, A. D. Kaminker, O. Y. Gnedin, P. Haensel, Phys. Rept. 354, 1 (2001).

[24] D. N. Spergel et al., Astrophys. J. Suppl. 148, 175 (2003)

[25] M. Fukugita and T. Yanagida, Phys. Lett. B174, 45 (1986).

[26] W. Buchmuller, R. D. Peccei and T. Yanagida, hep-ph/0502169

[27] W. Buchmuller, P. Di Bari and M. Plumacher, New J. Phys. 6,105 (2004); Annals Phys. 315 , 305 (2005); Nucl. Phys. B665, 445 (2003); W. Buchmuller, Acta Phys. Polon. B32 , 3707 (2001); Lect. Notes Phys. 616, 39 (2003); W. Buchmuller, R. D. Peccei and T. Yanagida, hep-ph/0502169

[28] D. Notzold and G. Raffelt, Nucl. Phys. B307, 924 (1988).

[29] J. C. D'Olivo, J. F. Nieves and M. Torres, Phys. Rev. D46, 1172 (1992); E. S. Tututi, M. Torres and J. C. D'Olivo, Phys. Rev. D66, 043001 (2002).

[30] R. Barbieri and A. Dolgov, Nucl. Phys. B 349, 743 (1991).

[31] K. Enqvist, K. Kainulainen and J. Maalampi, Nucl. Phys. B349, 754, (1991).

[32] G. Sigl and G. Raffelt, Nucl. Phys. B406, 423 (1993).

[33] P. Strack and A. Burrows, Phys.Rev. D71, 093004 (2005); P. Strack, hep-ph/0505056 (Master's Thesis).

[34] V. A. Kostelecky, J. Pantaleone and S. Samuel, Phys. Lett. B315, 46 (1993); V. Kostelecky and S. Samuel, Phys. Lett. B318, 127 (1993); V. Kostelecky and S. Samuel, Phys. Rev. D49, 1740 (1994); V. Kostelecky and S. Samuel, Phys. Rev. D52, 3184 (1995); S. Pastor, G.G. Raffelt, D.V. Semikoz, Phys.Rev. D65, 053011 (2002).

[35] J. C. D' Olivo and J. F. Nieves, Int. Jour. of Mod. Phys. A11, 141 (1996).

[36] J. Pantaleone, Phys. Lett. B287, 128 (1992), Phys. Rev. D46, 510 (1992).

[37] D. Boyanovsky and C. M. Ho, Phys. Rev. D69, 125012 (2004).

[38] D. Boyanovsky, Phys. Rev. D72, 033004 (2005).

[39] J. Schwinger, J. Math. Phys. 2, 407 (1961); K. T. Mahanthappa, Phys. Rev. 126, 329 (1962); P. M. Bakshi and K. T. Mahanthappa, J. Math. Phys. 41, 12 (1963); L. V. Keldysh, JETP 20, 1018 (1965); K. Chou, Z. Su, B. Hao And L. Yu, Phys. Rep. 118, 1 (1985); A. Niemi and G. Semenoff, Ann. of Phys. (NY) 152, 105 (1984); N. P. Landsmann and C. G. van Weert, Phys. Rep. 145, 141 (1987); E. Calzetta and B. L. Hu, Phys. Rev. D41, 495 (1990); ibid. D37, 2838 (1990); J. P. Paz, Phys. Rev. D41, 1054 (1990); ibid. D42, 529 (1990).

[40] R. D. Jordan, Phys. Rev. D33, 444 (1986).

[41] D. Boyanovsky, H. J. de Vega and R. Holman, Proceedings of the Second Paris Cosmology Colloquium, Observatoire de Paris, June 1994, pp. 127-215, H. J. de Vega and N. Sanchez, Editors World- Scientific, 1995; Advances in Astrofundamental Physics, Erice Chalonge Course, N. Sanchez and A. Zichichi Editors, World Scientific, 1995; D. Boyanovsky, H. J. de Vega, R. Holman, D.-S. Lee and A. Singh, Phys. Rev. D51, 4419 (1995); D. Boyanovsky, H. J. de Vega, R. Holman and J. Salgado, Phys. Rev. D54, 7570 (1996). D. Boyanovsky, H. J. de Vega, C. Destri, R. Holman and J. Salgado, Phys. Rev. D57, 7388 (1998).

[42] D. Boyanovsky, H. J. de Vega, R. Holman and D.-S. Lee, Phys. Rev. D52, 6805 (1995); (see also ref. 41]).

[43] S. Y.-Wang, D. Boyanovsky, H. J. de Vega, D.-S. Lee and Y. J. Ng, Phys. Rev. D61, 065004 (2000); D. Boyanovsky, H. J. de Vega, D.-S.Lee, Y.J. Ng and S.-Y. Wang, Phys. Rev. D59, 105001 (1999).

[44] M. E. Peskin and D. V. Schroeder, An introduction to Quantum Field Theory, Advanced Book Program, Addison-Wesley Pub. Co, Reading, Massachusetts, 1995.

[45] KamLAND Collaboration, K. Eguchi et al., Phys. Rev. Lett., 90, 021802 (2003); T. Araki et al., hep-ex/0406035

[46] L. Bento and F. C. Santos, Phys.Rev. D71, 096001(2005). 